\title{
Erschütterung der Wissenssysteme und methodische Neuansätze
}

Die Rekapitulation der Gattungsentwicklung im vorhergehenden Kapitel hat deutlich gemacht, wie wurzelhaft die Auseinandersetzung mit dem Epos in der Moderne jeweils mit dem Befund und der Überwindung von systemübergreifenden Krisen verbunden ist. Und gleichzeitig hat sich abgezeichnet, dass die Erschütterungen der Epochenschwelle nicht nur die sozialen und politischen Strukturen, sondern ebenso die ästhetischen und kulturellen Programme betreffen. Inwiefern das gerade auch für die Gattungspoetologie als Leitsystem der Philologie und insbesondere für das Eposkonzept gilt, wurde gleichfalls an Goethes frühen epischen Projekten anschaulich, die bereits auf eine offensichtliche Lust am Ausprobieren und am Vermischen verschiedener Formen verweisen. Diese Experimentierfreude steht seltsam quer zur kunstrichterlichen Strenge, die Goethe zu diesem Zeitpunkt attestiert wird sowie zum minimalistisch-reduzierten Model der Gattungstrias, das für diese vermeintliche Hochphase klassizistischer Kunsttheorie als selbstverständlich gilt. Die traditionelle Forschung konstatiert gemeinhin erst für 1805 - also unauflöslich mit dem Tod Schillers verbunden - eine genuine Krisenerfahrung im Klassizismus, die sich sowohl in Goethes nun konsequent betriebener Historisierung als auch seiner grundsätzlichen Hinwendung zu Problemen der Form ausdrücke. ${ }^{1}$ Die Jahre davor gelten als Höhepunkt der klassizistischen Kultur in Weimar - frühen historisierenden Bemühungen und sich bemerkbarmachendem Geltungsverlust normativer Prämissen wird allenfalls eine marginale Bedeutung zugestanden. Für gewöhnlich werden sie aber ebenfalls konsequent in den Kontext einer normorientierten Kunsttheorie gestellt.

Die hier skizzierte ambivalente Gleichzeitigkeit von rigider Gattungspoetologie und Vermischung der Formen lässt allerdings erahnen, dass die Gestaltung und Benennung von literarischen Werken nach normativen

1 Vgl. Keller: Lebendiger Abglanz. Goethes Italien-Projekt als Kulturanalyse. S. 80; Grave, Johannes: Der „ideale Kunstkörper“. Johann Wolfgang Goethe als Sammler von Druckgraphiken und Zeichnungen (= Ästhetik um 180o, Bd. 4). Göttingen:Vandenhoeck \& Ruprecht 2006. S. 132 und Einem, Herbert von: Ein ungedrucktes Manuskript Johann Heinrich Meyers über Michelangelo. In: Goethe Jahrbuch 94 (1977). S. 256-285, hier S. 264. 
Kriterien bereits zu diesem Zeitpunkt problematisch geworden ist und die Frage, wie mit diesem Legitimationsverlust umgegangen werden soll, nun verstärkt in den Fokus rückt. Es ist deshalb eine wichtige Erkenntnis der jüngsten Forschung, dass der vermeintlich monophone Klassizismus tatsächlich bereits im letzten turbulenten Jahrzehnt vor der Jahrhundertwende in eine kritische Phase eintritt und seine konservativen ästhetischen Programme in Bedrängnis geraten. ${ }^{2}$

Unter dem Problemdruck der frühen Moderne findet im ästhetischen und kunsttheoretischen Diskurszusammenhang bereits zur Zeit der Eposdebatte eine Aufmerksamkeitsverschiebung auf die miteinander in Beziehung stehenden Prinzipien von Bewegung und Zeitlichkeit statt. Dieses Bewusstwerden von Dynamisierung und Verzeitlichung lässt sich gerade an den für die Dichtung zentralen Bezugssystemen der Kultur und der Sprache besonders augenfällig als dialektische Verschränkung von Zerfallsdiagnose und der Suche nach adäquaten d.h. reflexiven methodischen Neuansätzen beobachten. An Goethes kritischer Betrachtung der Kultur zum einen und seinen Überlegungen zur Morphologie zum anderen soll deshalb präzisierend gezeigt werden, wie die darin aufgeworfenen methodischen Problemstellungen und vor allem die sich daraus ergebenden Chancen der Neuerung bereits in den frühen 179oer Jahren zu einem grundsätzlich veränderten Formbewusstsein führen. Im Weiteren soll Goethes morphologisches Formverständnis nicht nur als konstitutiv für die Erneuerung des Epischen jenseits der konservativen Gattungspoetologie betrachtet werden, sondern es soll darüber hinaus auch aufgezeigt werden, wie er aus der Auseinandersetzung mit dem Epos gerade auch entscheidende Anregungen für sein Formdenken zurückerhält. Dies liegt nicht allzu fern, entstehen doch die Gedanken zur Morphologie nicht zuletzt auf dem Hintergrund einer Erzählkrise, die sich in Abbruch und Wiederaufnahme des Wilhelm Meister-Romans sowie der zeitgleichen Beschäftigung mit anderen Formen des Erzählens wie der Novelle, der Idylle und besonders dem Epos offenbart. Im Folgenden wird die Morphologie gemäß Goethes Anspruch, sie für alle Wissensbereiche fruchtbar zu machen, als universale Methode verstanden, die sich gerade auf derartige Wechselwirkung richtet, anstatt auf rigide, abgeschlossene Systeme.

Der sich im Kontext von Goethes morphologischen Überlegungen als zentral erweisende Begriff des Experiments, so viel sei vorweggenommen, entspricht als fragile, dynamisch-fragmentarische Verfahrens- und

2 Vgl. Ehrmann, Daniel; Wolf, Norbert Christian (Hrsg.): Klassizismus in Aktion. Goethes Propyläen und das Weimarer Kunstprogramm (= Literaturgeschichte in Studien und Quellen, Bd. 24) Wien, Köln, Weimar: Böhlau 2016. 
Darstellungsform ganz dem Anliegen, sich wandelnde, lebendige und bisweilen prekäre Beziehungen zu beobachten und zu beschreiben. Durch ihn eröffnet sich Goethe ein alternatives Instrumentarium, das es ermöglicht, der Dynamik, Komplexität und Verzeitlichung der Wissenssysteme - und damit auch des Gattungssystems - gerecht zu werden, indem sie selbst als dynamische und komplexe Felder in ständiger gegenseitiger Wechselwirkung verstanden werden. Aus dieser Perspektive kann die zwischen Verabschiedung und Restitution hin- und her schwankende Eposdebatte der Epochenschwelle als Verschiebung weg von der normativen Gattung des Epos hin zum Epischen als Reflexionsmedium beleuchtet werden. Die darin sichtbar werdende Prekarität betrifft sowohl das Epos als gefährdete Form der Literatur als auch die Kunst und ihre Produktionsbedingungen überhaupt. Gleichzeitig werden diese Brüche reflexiv aufgearbeitet, so dass das Epische als ambivalentes, experimentelles Kunsterneuerungsprojekt der kulturellen Moderne erscheint.

Mit dieser Neuperspektivierung der Epochenschwelle und der darin stattfindenden Eposdebatte unter dem Eindruck von Goethes morphologischem Formdenken werden somit gleich mehrere etablierte Forschungspositionen revidiert: Die Zäsur von 1805 als Ende des sich in Weimar konzentrierten Klassizismus und Beginn von Goethes Spätwerk wird fragwürdig. Vielmehr scheinen Beunruhigung, Krisendiagnose und Öffnung gegenüber nichtnormativen Konzepten bereits vor der klassizistischen Hochphase virulent zu werden und den Klassizismus geradezu als komplementäre Ästhetik und Kunsttheorie anzutreiben. Daraus resultiert eine veränderte Perspektive auf das Epochenbild der Klassik, die an die von Claudia Keller vorgestellte Idee eines Klassizimus im Experiment ${ }^{3}$ anschließt und auch auf die Phasendatierung von Goethes Werk.

\subsection{Die Unruhe in der Kultur}

Schon die Reise nach Italien zwischen 1786 und 1788, die Schulz bezeichnenderweise als "Flucht" beschreibt, ${ }^{4}$ ist von einer persönlichen Krisenerfahrung motiviert, deren problematischen Kern Goethe bereits zu diesem Zeitpunkt

3 Vgl. Keller, Claudia: Die ungeschriebenen Propyläen - Klassizismus im Experiment. In: Ehrmann; Wolf (Hrsg.): Klassizismus in Aktion. Goethes Propyläen und das Weimarer Kunstprogramm. S. $387-407$.

4 Schulz, Karlheinz: Wandlungen und Konstanten in Goethes Ästhetik und literarischer Laufbahn. Goethezeitportal. http://www.goethezeitportal.de/fileadmin/PDF/db/wiss/goethe/ schulz_wandlungen_konstanten.pdf; Erstpublikation: 11.5.2010 [abgerufen am 20.12.2016]. 
als eine tief empfundene Beunruhigung mit ästhetischem Endzeitcharakter darstellt:

Auf dieser Reise, hoff ich, will ich mein Gemüth [sic] über die schönen Künste beruhigen, ihr heilig Bild mir recht in die Seele prägen und zum stillen Genuss bewahren. Dann aber mich zu den Handwerkern wenden, und wenn ich zurückkomme, Chymie [sic] und Mechanik studieren. Denn die Zeit des Schönen ist vorbei, nur die Not und das strenge Bedürfnis erfordern unsre Tage. [Hervorhebung M.E. $]^{5}$

Diese Hoffnung, das Gemüt durch eine bewusst auf Entschleunigung, ja sogar konservierenden Stillstand ausgerichtete Kunstrezeption zu beruhigen und zu stabilisieren, zerschlägt sich allerdings. Die Bereisung Italiens vermag an Goethes kulturkritischem Pessimismus nichts zu ändern, im Gegenteil: Spätestens nach seiner Rückkehr in das „prosaische“6 ${ }^{4}$ Deutschland werden ihm die hier vorherrschenden, problematischen Zersetzungstendenzen erneut drastisch vor Augen geführt: „Aus Italien dem Formreichen war ich in das gestaltlose Deutschland zurückgewiesen" beklagt sich Goethe bei Knebel und wundert sich, dass hier überhaupt "noch ein Wölkchen Poesie über meinem Scheitel schweben bleibt." ${ }^{\text {"7 }}$ Diese auffällige Begriffsverwendung von Form und Gestalt deutet auf die intensive Beschäftigung mit Fragen nach dem Ursprung der Form und ihrer Gestaltwerdung in den späten 179oer Jahre sowie die in der Einleitung in die Propyläen konstatierte „Schwierigkeit, von dem Formlosen zur Gestalt überzugehen “8 voraus. Bereits jetzt lassen sich Konsequenzen in Goethes Denken beobachten, die sich aus einer vorrevolutionären Unruhe speisen: Zum einen richtet sich sein Interesse nun entschieden auf die Kultur, ihre Entwicklungsmöglichkeiten und die offenkundig fragwürdig gewordene kulturelle Leistungsfähigkeit der Formen der Kunst. Und zum anderen sind es die organischen Kräfte der Natur sowie die Suche nach einer adäquaten wissenschaftlichen Beschreibung der hier beobachteten Transformationsprozesse, die in den Blick rücken. Dabei gelangt Goethe zunehmend zu der Einsicht, dass das im zitierten Brief an Frau von Stein aus dem Jahre 1786 noch aufscheinende Konservierungs-Programm - und damit auch das klassizistische Mimesis-Ideal - keine adäquaten Problemlösungen mehr bieten. Präsentieren sich Natur- und Kulturbetrachtung, Wissenschaft und Ästhetik in der Folge der Italienreise doch immer mehr als ein komplexer,

5 Goethe an Frau von Stein, 5.10.1786, MA 3.1. S. 107.

6 Goethe an Knebel, 9.7.179o, MA 4.2. S. 1019.

7 HA 13. S. 101.

8 MA 6.2. S. 967. 
dynamischer Wissenszusammenhang, in dem Erkenntnisse und Erkenntnisstrategien von einem Feld auf das andere transferiert werden können.

Auch der Italien-Aufenthalt, der sich explizit der Beschreibung der italienischen Natur und Kultur im Zusammenhang mit exemplarischen Betrachtungen der antiken Kunst widmet, steht im Zeichen des bereits in den ersten Weimarer Jahren diagnostizierten Kulturzerfalls. Darauf verweist Goethe selbst explizit, wenn er in Schicksal der Handschrift rückblickend beschreibt, wie er während seiner Reisen durch Italien neben Studien zur griechischen Kunst und zu den treibenden Kräften der Natur auch die „Sitten der Völker“ - also die Kultur - untersucht und an ihnen gelernt hat, „wie aus dem Zusammentreffen von Notwendigkeit und Willkür, von Antrieb und Wollen, von Bewegung und Widerstand ein Drittes hervorgeht, was weder Kunst noch Natur, sondern beides zugleich ist, notwendig und zufällig, absichtlich und blind; ich verstehe die menschliche Gesellschaft. [Hervorhebung M.E.] ${ }^{\text {a9 }}$ Damit wird zum einen der strukturelle Zusammenhang von Natur, Kunst und Gesellschaft dezidiert als ein dynamischer und spezifisch zeitlich verfasster ausgewiesen und zum anderen wird die Gesellschaft als eine explizit kulturell geprägte, dritte Kategorie zwischen Kunst und Natur determiniert. ${ }^{10}$

Auf den lateinischen Wortstamm zurückgeführt, ist der Begriff der Kultur zunächst gleichbedeutende mit Agrikultur bzw. Ackerbau - also der Bearbeitung des Bodens - und wird im deutschen Sprachraum bis ins 18. Jahrhundert auch vornehmlich in dieser Weise verwendet.1 Etwa ab $175^{\circ}$ wird dann die Bedeutung der Pflege, Bearbeitung und Nutzbarmachung sukzessive auf alle möglichen Gesellschaftsbereiche übertragen - eine Entwicklung, die sich in Deutschland durch die Beschäftigung mit der französischen Aufklärung durchzusetzen beginnt. Sie lässt sich also zum Beispiel auch zunehmend metaphorisch auf den Körper, den Geist, das menschliche Handeln, die Ernährung, den Städte- und Häuserbau anwenden. Dieser sich zu Beginn der Moderne transformierende Begriff von Kultur beschreibt dann sämtliche Bemühungen des Menschen, sich und seine Umgebung durch Handwerk, Wissenschaft und nicht zuletzt auch die Kunst zu bilden - sprich zum Besseren voranzutreiben. In Herders geschichtsphilosophischer Abhandlung Ideen zur

9 HA 13. S. 101.

10 Vgl. Puszkar, Norbert: Goethes Volksbegriff und Habermas' Begriff der „Lebenswelt“: Die Kultur der norditalienischen Städte in der Italienischen Reise. In: German Studies Review 1 (2007). S. $75^{-96}$, hier S. 76.

11 Zum Kulturbegriff vgl. Andreas Preussner: Kultur. In: Wörterbuch der Philosophie (online). http://www.philosophie-woerterbuch.de [abgerufen am 2.2.2017] und Heijl, Peter M.: Kultur. In: Nünning, Ansgar (Hrsg.): Metzler Lexikon Literatur- und Kulturgeschichte. Ansätze - Personen - Grundbegriffe. S. 391-393. 
Philosophie der Geschichte der Menschheit (1784-1791) werden diese modernen Bedeutungsdimensionen zum ersten Mal ausgebreitet und Kultur als ein komplexes Gebilde betrachtet, das jeweils unter verschiedenen historischen und geographischen Bedingungen seine spezifische Ausprägung erhält. Zum einen lassen sich so auch verschiedene Kulturen miteinander vergleichen, kulturelle Gemeinsamkeiten und Unterschiede zwischen Völkern können herausgestellt und gegeneinander abgewogen werden. Und zum anderen lässt sich so nach dem Ursprung und der Entwicklung von Kultur fragen, nach den Ordnungsprinzipien ihrer Ausprägungen über die Zeit hinweg. Auf diese Abfolgeverhältnisse und Übergänge zwischen den Kulturen bzw. ihren Entwicklungsstufen richtet Herder sein Augenmerk und begreift sie als einen national geprägten, linearen Fortschrittsprozess, in dem sich Vernunft und Humanität immer weiter entfalten. ${ }^{12}$

Damit wird deutlich, inwiefern der Kulturbegriff an der Epochenschwelle um 1800 sowohl mit dem bürgerlichen Bildungsideal wie auch mit einer grundsätzlich positiven Fortschritts-Vorstellung eng verbunden ist und in sich selbst bereits eine Entwicklung von der Natur bzw. vom Boden hin zu geistigen Gefilden, zur Kunst nachvollzieht. Mit dem Fortschritt ist ein Moment der Steigerung verbunden, an das sich einerseits das Ziel einer stetigen Optimierung des Menschen knüpfen lässt, das andererseits aber auch mit Bewegung, Beunruhigung und letztlich Diskontinuität verschränkt ist. ${ }^{13}$ Der daran anschließende, bereits im 17. Jahrhundert durch Samuel von Pufendorf eingeführte, wertende Gebrauch von Kultur als Gegenbegriff zu Naturzustand und Barbarei wird dann für den normorientierten Kulturbegriff der Epochenschwelle besonders ausschlaggebend. ${ }^{14}$ Im 18 . Jahrhundert erhält der Kulturbegriff zunehmend eine geschichtsphilosophische Bedeutung. Er soll die historische Entwicklung der Menschheit bzw. ihrer Kultur nachzuvollziehbar machen und wird gleichzeitig komplexer und diversifizierter: Er beschreibt nun sowohl den Kulturalisierungs-Prozess als ein Verfahren mit spezifischen Bedingungen als auch thematisch die einzelnen Ereignisse. Zudem wird Kultur als Bildungskonzept vom aufstrebenden Bürgertum als soziales Distinktionsmerkmal gegenüber dem „degenerierten“ Adel und der "primitiven“

12 Zu Herders Kulturphilosophie vgl. Kopp, Bernhard: Beiträge zur Kulturphilosophie der deutschen Klassik. Eine Untersuchung im Zusammenhang mit dem Bedeutungswandel des Wortes Kultur (= Monographie zur philosophischen Forschung, Bd. 128). Meisenheim am Glan: Hain 1974. S. 19-26.

13 Vgl. Konersmann, Ralf: Wörterbuch der Unruhe. Frankfurt a. M.: Fischer 2017. S. 112-116.

14 Vgl. Heijl: Kultur. S. 391-393, hier S. 391. 
Unterschicht reklamiert. ${ }^{15}$ Mit diesen Veränderungen im Kulturbegriff entsteht nicht zuletzt auch die Möglichkeit der Reflexion, die die Spannungen zwischen einem endlosen, der Steigerung verschriebenen Transformationsprozess und der Hoffnung auf Beruhigung und Stabilisierung zu überdenken oder zumindest auszustellen vermag. ${ }^{16}$ Wenn Goethe das Verhältnis von Natur und Kultur, Mensch und Kunst nämlich gerade nicht als lineare Abfolge, sondern als „beides zugleich“17 beschreibt, widerspricht das nicht nur dem damals geltenden normativen Kulturbegriff, sondern es wirft vor allem auch ein kritisches Licht auf den ihm eingeschriebenen Fortschrittsglauben und das damit verbundene Prinzip der Beschleunigung.

$\mathrm{Zu}$ Beginn von Goethes kulturanalytisch motiviertem Italien-Aufenthalt kristallisieren sich unter den vielen Eindrücken der norditalienischen Städte vor allem zwei Interessensgegenstände heraus: Die Wiederbelebung der antiken Architektur und das italienische Volk. Letzeres rückt im Laufe der Reise sogar immer mehr ins Zentrum seiner Aufmerksamkeit und zwar nicht bloss als „Agent kurioser Sitten und Bräuche oder als Inbegriff einer mediterranen Lebensfreude“, wie Puszkar schreibt, sondern gleichfalls als „Beispiel und Vorbild einer Kommunikationskultur.“18 In Venedig scheinen ihm zunächst alle kulturellen Manifestation marginalisiert: „alles [ist] gesagt und gedruckt.“ Die „Haupt Idee“, die sich Goethe hier aber „aufdringt, ist wieder [das] Volk. Große Masse! und ein notwendiges unwillkürliches Dasein“. ${ }^{19}$ Dieses Volk und vor allem seine soziale Praxis ziehen den deutschen Dichter völlig in ihren

15 Vgl. Sommer, Roy: Kulturbegriff. In: Nünning, Ansgar (Hrsg.): Metzler Lexikon Literaturund Kulturgeschichte. Ansätze - Personen - Grundbegriffe. S. 395-396.

16 Vgl. Böhme, Hartmut; Matussek, Peter et al. (Hrsg.): Kulturwissenschaft Orientierung. Was sie kann, was sie will. Reinbek b. Hamburg: Rowohlt 2002. S. 106. Diese diskursive Erweiterung und Vervielfältigung des Kulturbegriffs wird sich dann im 19. Jahrhundert mit zunehmender Geschwindigkeit weiter fortsetzen - aus Kultur werden Kulturen und in dieser Modifizierung wird Kultur nun zunehmend auf das große Ganze von Geschichte, Wissen und Kunst einer Nation bezogen. Daraus ergibt sich ein immer unübersichtlicheres und heterogeneres Gebilde von verschiedenen, nebeneinanderstehenden Kulturen mit unterschiedlichen Kulturalisierungsprozessen und -bedingungen. Dieser zunehmend als problematisch wahrgenommenen Pluralisierungsentwicklung setzt Hegel spätestens ab 1820 wirkungsmächtig eine modellhafte Vorstellung von klar unterscheidbaren, sich ablösenden Kulturstufen entgegen, die darauf ausgerichtet ist, eine geregelte, berechenbare Transformationslogik und eine überschaubare Prozessualität der Kulturgeschichte zu bewahren.

17 HA 13. S. 101.

18 Puszkar: Goethes Volksbegriff und Habermas' Begriff der „Lebenswelt“: Die Kultur der norditalienischen Städte in der Italienischen Reise. S. $77 \mathrm{ff}$.

19 Tagebucheintrag vom 29.9.1786, MA 3.1. S. 9 o. 
Bann und im venezanischen Theater offenbart sich Goethe dann auch die systemische Relevanz des Volkes für die Kunst:

Doch ist immer wieder das Volk die Base worauf das alles steht. Das Ganze machts, nicht das einzelne. Auf dem Platz und am Ufer und auf den Gondeln und im Palast. Der Käufer und Verkäufer, der Bettler der Schiffer die Nachbarin, der Advokate und sein Gegner alles lebt und treibt und lässt sichs angelegen sein und spricht und beteuert und schreit und bietet aus und singt und schilt und flucht und lärmt. Und abends gehn sie ins Theater und sehn und hören das Leben ihres Tags, nur künstlich zusammengestellt, artiger aufgestutzt mit Märgen durchflochten etc. und freuen sich kindisch und schreien wieder und klatschen und lärmen. ${ }^{20}$

Die Beobachtung der Italiener und ihrer aktiv gelebten Kultur führt Goethe auf die zentralen Begriffe von Öffentlichkeit, Nation und Sprechen, die sich dann auch für sein Epos-Projekt als ausschlaggebend erweisen werden. Noch am selben Abend notiert er:

Ich habe nun öffentlich reden hören:

1) 3 Kerls auf dem Platz nach ihrer Art Geschichten erzählend.

2) 2 Prediger

3) 2 Sachwalter

4) Die Komödianten, besonders den Pantolon.

alle haben etwas gemeines, sowohl weil sie von Einer Nation sind, die beständig im Leben und sprechen begriffen ist, als auch weil sie sich unter einander nachahmen. Sie haben gewisse Lieblings Gesten, die ich mir merken will, und überhaupt üb' ich mich sie nachzumachen und will euch in dieser Art Geschichten erzählen, wenn ich zurückkomme ob sie gleich mit der Sprache vieles von ihrer Originalität verlieren $[\ldots] .{ }^{21}$

Diese in Italien erlangte, an Herder anschließende Auffassung vom Volk als Träger einer national begrenzten Kulturgemeinschaft spielt für Goethes Suche nach Möglichkeiten, den konstatierten Bruch der Kulturtradition zu überwinden, eine zentrale Rolle. Stärker als bei Herder steht Goethes Volksbegriff jedoch in enger Verbindung mit der Vorstellung der menschlichen Gesellschaft als lebendiger, interagierender Gemeinschaft der Notwendigkeit, durch deren mündlichen Austausch Öffentlichkeit und eine kollektive Identität entstehen. Mit Ausnahme von Georg Simmels Kulturphilosophie unterscheidet sich Goethes Konzept allerdings erheblich von anderen zeitgenössischen und erst

20 Tagebucheintrag vom 4.10.1786, MA 3.1. S. 103. f.

21 Ebd. S. 106. 
recht späteren Volksbegriffen sowie damit verbundenen Kulturmodellen. ${ }^{22}$ In der Verhandlung seines alternativen, auf Kontinuität ausgerichteten Kulturbegriffs bezieht sich Goethe nun an entscheidender Stelle spezifisch auf das Epos. So ist es gerade Homers Odyssee, in der er die Gleichzeitigkeit von Natur und Kunst als Kriterium einer lebendigen Kultur erkennt, die mit Böhme gesprochen „die Sicherung von räumlicher Ständigkeit und zeitlicher Stetigkeit“23 gewährleisten soll. „Was den Homer betrifft", schreibt Goethe an Herder aus Neapel im Mai 1787,

ist mir wie eine Decke von den Augen gefallen. Die Beschreibung, die Gleichnisse, etc. kommen uns poetisch vor und sind doch unsäglich natürlich, aber freilich mit einer Reinheit und Innigkeit gezeichnet, vor der man erschrickt. Selbst die sonderbarsten erlogenen Begebenheiten haben eine Natürlichkeit, die ich nie so gefühlt habe als in der Nähe der beschriebenen Gegenstände. [...] Nun ich alle diese Küsten und Vorgebirge, [...] Klippen und Bänke und das alles umgebende Meer mit so vielen Abwechselungen und Mannigfaltigkeiten im Geiste gegenwärtig habe, nun ist mir erst die Odyssee ein lebendiges Wort. [Hervorhebung M.E. $]^{24}$

Die Überzeugung von Homers Werk als Inbegriff einer lebendigen Kultur steht zu diesem Zeitpunkt noch im Zeichen des klassizistischen HarmonieGedankens und der von Winckelmann inspirierten Idealisierung der griechischen Antike. Unterschwellig wird hier aber in der geologischen Beschreibung der Mittelmeerlandschaft mit Bezug zum Epos bereits die ästhetische Idee eines gleichzeitigen Nebeneinanders widersprüchlicher Prinzipien angedacht. Darauf lenkt auch Claudia Keller den Blick, wenn sie in ihrer Dissertation zum Italienprojekt einsehbar macht, wie Goethes Überlegungen zu Bedingungen und Möglichkeiten der Darstellung seit Mitte der 178oer Jahren in enger Verbindung stehen mit grundsätzlichen Fragen zur Kultur, ihrem Verhältnis zur Natur und ihrer modernespezifischen Dynamisierung, die im Begriff des Lebendigen positiv problematisiert wird. ${ }^{25}$ Goethes Kulturbegriff bestimmt sich eindeutig morphologisch - er betrachtet das Volk, die es umgebende Natur und die von ihm hervorgebrachte Kunst als interagierende, sich gegenseitig

22 Vgl. Faath, Ute: Mehr-als-Kunst. Zur Kunstphilosophie Georg Simmels. Univ. Karlsruhe 1998 [Dissertationsschrift].

23 Böhme, Hartmuth: Vom Cultus zur Kultur(wissenschaft). Zur historischen Semantik des Kulturbegriffs. In: Glaser, Renate; Luserke, Matthias (Hrsg.): Kulturwissenschaft Literaturwissenschaft. Positionen, Themen, Perspektiven. Opladen: Westdeutscher Verlag 1996. S. 48-68, hier S. 53 .

24 Goethe an Herder, 17.5.1787, HA 11. S. 323.

25 Vgl. Keller: Lebendiger Abglanz. Goethes Italien-Projekt als Kulturanalyse. S. 58. 
bedingende Manifestationen der einen inneren Struktur ${ }^{26}$ - und lenkt so die Aufmerksamkeit bereits früh auf die Phänomene von Bewegung und Vielheit. Damit ist auch der Übergang zur Morphologie als Erklärungsmuster für das Epos und darüber hinaus für die nun immer drängenderen Fragen der Gattungspoetik angedeutet.

\subsection{Mediale Beschleunigung und die Verzeitlichung der Form}

Die Ausführungen zu Goethes Italien-Aufenthalt haben offenbart, dass sich kulturelle Verunsicherung und Beunruhigung schon vor der historischen Zäsur der Französischen Revolution deutlich bemerkbar machen. Wie im Folgenden aber aufgezeigt werden soll, bestätigt die erschreckende Revolutionserfahrung die befürchtete Auflösung bisheriger Strukturen und erlangt insofern die funktionale Wirkung eines Katalysators für die Akteure der Epochenschwelle. So muss etwa Goethe sein Vorhaben, den Kulturraum Italiens in seiner abgeschlossenen Ganzheit darzustellen, nicht zuletzt auch aufgrund der Napoleonischen Kriege aufgeben. ${ }^{27}$ „Was wir aus diesem allgemeinen und besonderen Schiffbruch retten, magst du, wenn es dich interessiert, aus den Propyläen von Zeit zu Zeit ersehen“28, wird er später rückblickend an Heinrich Jacobi schreiben. In dieser Diagnose zum Scheitern des Italienprojekts schließen sich die Erfahrung radikaler historischer Veränderung und eines allgemeinen Einbruchs von Dynamik zur Metapher des Schiffbruchs zusammen. Mit der plötzlichen Eskalation des politischen Ermächtigungsprozesses in Frankreich droht nicht nur ein beispielloser Bruch der tradierten Gesellschaftsstrukturen, sondern ist der kontinuierliche Fortgang der abendländischen Kulturgeschichte grundsätzlich in Frage gestellt. ${ }^{29}$ Nur Bruchstücke - von der italienischen Kultur aber auch von der Kulturgeschichte an sich - können überhaupt noch "gerettet" werden. ${ }^{30}$ Dass die „Zeit des Schönen“31 damit nun

26 Vgl. Puszkar: Goethes Volksbegriff und Habermas’ Begriff der „Lebenswelt“: Die Kultur der norditalienischen Städte in der Italienischen Reise. S. 77.

27 Für eine umfassende Beschreibung des Italienprojekts vgl. Baum, Richard: Der Genius Italiens - Goethes dritte Reise in den Süden als Wendepunkt im Schaffensprozess. In: Hirdt, Willi; Tappert, Birgit (Hrsg.): Goethe und Italien. Bonn: Bouvier 20o1. S. 1-55.

28 FA II, 5. S. 11.

29 Vgl. Oschmann, Dirk: Das Epos in Zeiten des Romans. Goethes Hermann und Dorothea. S. 167-189, hier S. 168.

30 Vgl. dazu auch Keller, Claudia: Aus dem Schiffbruch gerettet? Kulturhistorische Zeitreflexion der ,Weimarer Kunstfreunde؛. In: Goethe Jahrbuch 131 (2014). S. 51-58.

31 Goethe an Frau von Stein, 5.10.1786, MA 3.1. S. 107. 
endgültig vorbei ist, bezeugt auch der einsetzende Geltungsverlust normativer Prämissen zugunsten autonomieästhetischer Konzepte.

\subsubsection{Die Revolution als Katalysator für den medialen Wandel}

Dass sich keine Beruhigung einzustellen vermag, hat also durchaus mit dem Ausbruch der Revolution zu tun und, wie es nun zu zeigen gilt, mit der gleichzeitigen Entstehung einer frühen Form der Informationsgesellschaft. Neue Publikationstechniken führen seit den 1770er Jahren in ganz Europa zu einer rapide anwachsenden Zahl von Zeitungen, Zeitschriften sowie Einzelpublikationen. Diese sich explosionsartig entfaltende Presse bildet die Voraussetzung für die im Zeichen der Revolution entstehenden medialen Veränderungen. ${ }^{32}$ Mit dem Sturm der Bastille wird die Französische Revolution im Juli 1789 zum internationalen Medienereignis. Gerade die sensationellen Pariser journées révolutionnaires bilden überall ein dominantes Nachrichtenthema und revolutionäre Texte, Bilder und Hymnen werden in grossem Stil weit über die französischen Grenzen hinaus exportiert. Nirgendwo ist der multimediale Transfer der Neuigkeiten und der Revolutionskultur aus Frankreich allerdings unmittelbarer, dichter und kontinuierlicher als im absolutistischen Deutschland. Die Ereignisse des Jahres 1789 stellen für die deutsche Öffentlichkeit ein bis dahin nicht gekanntes Spektakel dar. Täglich überschwemmen zahlreiche, neue, sich teilweise konkurrierende Informationen das lesefähige deutsche Publikum ${ }^{33}$ und führen die seit der Aufklärung stattfindende Verschriftlichung des Wissens, der Wissensbildung und der Wissenstradierung auf einen neuen Höhepunkt.

Dazu äußert sich der Schriftsteller Heinrich Christian Boie bereits im Dezember 1789 kritisch: Es sei überflüssig, die revolutionären Ereignisse in Frankreich zu schildern, „da Deutschland mit Schriften darüber bis zum Eckel

$3^{2} \mathrm{Zu}$ den Zusammenhängen von Französischer Revolution, publizistischer Innovation und Revolutionswahrnehmung in Deutschland vgl. Voss (Hrsg.): Deutschland und die Französische Revolution; Reichardt, Rolf: Die Französische Revolution und Deutschland. Thesen für einen komparatistischen kulturhistorischen Neuansatz. In: Aretin, Karl Otmar Freiherr von; Härter, Karl (Hrsg.): Revolution und konservatives Beharren. Das Alte Reich und die Französische Revolution (= Veröffentlichungen des Instituts für europäische Geschichte Mainz, Abteilung Universalgeschichte, Beiheft 32). Mainz: von Zabern 199o. S. 21-28; Schumann, Axel: Berliner Presse und Französische Revolution. Das Spektrum der Meinungen unter preussischer Zensur 1789-1806. Berlin: Univ. Berlin 2001 [Dissertationsschrift]. Und http://ieg-ego.eu/de/threads/europaeische-medien/ europaeische-medienereignisse/rolf-reichardt-die-franzoesische-revolution-alseuropaeisches-medienereignis-1789-1799 [abgerufen am 8.6.2018].

33 Vgl. Vierhaus, Rudolf: „Sie und nicht Wir“. Deutsche Urteile über den Ausbruch der Französischen Revolution. S. 1-15, hier S. 7 . 
überschwemmt “34 werde. Inwiefern in der Revolution, aber besonders auch in der forcierten Berichterstattung darüber, eine fundamentale Überforderungserfahrung offenbar wird, belegt auch die bekannte Aussage des Hamburger Journalisten Johann Wilhelm von Archenholz: „Die Französische Revolution“, schreibt er 1789, „verdrängt durch ihr gewaltiges Interesse alles; die besten Gedichte bleiben ungelesen. Man greift nur noch nach Zeitungen und solchen Schriften, die den politischen Heisshunger stillen“. ${ }^{35}$ Damit wird nicht nur der mediale Wandel der öffentlichen Kommunikation weg von der mündlichen hin zu einer schriftlichen Massenkommunikation thematisiert, sondern spezifisch auch auf die Verdrängung des Fiktiven durch das Faktische hingewiesen, die im 19. Jahrhundert die allgemeine Verschiebung weg von ästhetischen, hin zu empirischen Erkenntnismodellen begleitet.

Die stete Überholung der Berichterstattung durch die Ereignisse und eine dadurch immer verspätete, bereits bei ihrem Erscheinen veraltete Reaktion ${ }^{36}$ kommentiert auch Goethe während seiner Arbeit an Reineke Fuchs hellsichtig: „Der Dichter konnte der anrollenden Weltgeschichte nicht nacheilen und musste den Abschluss sich und anderen schuldig bleiben, da er das Rätsel

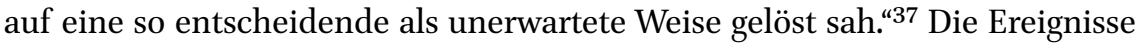
der Erfahrungswelt sowie ihre beschleunigte Abfolge zu erfassen und zu vermitteln, wird hier als spezifisches Unvermögen der Dichtung ausgewiesen und führt insofern auf die Konsequenzen der Dynamisierung und Ökonomisierung der Sprache für die Dichtung, deren Medium und Material sie ist: Verschriftlichung und Faktisierung führen zu einer Beschleunigung der Zeichenabfolge, die Zuständigkeiten und medialen Geltungszuschreibungen der literarischen Formen müssen deshalb neu ausgehandelt werden. ${ }^{38}$ Dass dabei der Frage nach der medialen Repräsentation von Erzählen besondere Aufmerksamkeit zukommt, dürfte kaum überraschen, konkurrenzieren die neuen periodischen Informationsmedien doch vor allem die traditionellen Formen

34 [Anonymus]: Ueber Voltairens Vorhersagung, 1789. S. 636 f., zitiert nach http://ieg-ego. eu/de/threads/europaeische-medien/europaeische-medienereignisse/rolf-reichardtdie-franzoesische-revolution-als-europaeisches-medienereignis-1789-1799\#MedialerTransferderRevolution [abgerufen am 8.6.2018].

35 Archenholz, Johann Wilhelm von: Minerva. Ein Journal historischen und politischen Inhalts, hrsg. v. dems., vormals Hauptmann in Königl. Preußischen Diensten, Bd. 7. Jena: Bran 1793. S. 199.

$36 \quad$ Vgl. MA 4.1. S. 920.

37 MA 14. S. 513 .

38 Vgl. Vellusig, Robert: Verschriftlichung des Erzählens. Medienprobleme des Romans im 17. und 18. Jahrhundert. In: Internationales Archiv für Sozialgeschichte der deutschen Literatur 1 (2005). S. 55-99, hier S. 57 und Geyer; Lehmann: Aktualität. Zur Geschichte literarischer Gegenwartsbezüge und zur Verzeitlichung der Gegenwart um 180o. S. 33-56. 
der mündlichen Überlieferung. Während der prosaische Roman jetzt als die Möglichkeit schlechthin erscheint, ein Erzählen zu gestalten, das den kognitiven und ästhetischen Potenzialen der medialen Ausprägung der Schriftlichkeit gerecht wird, verliert das Epische mit seiner Ausrichtung auf Oralität und eine poetisch verdichtete Sprache an Bedeutung. Inwiefern die Annäherung des Erzählens an den modernen Sprachgebrauch aber auch eine Loslösung aus kulturrelevanten Kontexten bedeutet und insofern mit dem Verlust einer ganz grundsätzlichen Vermittlungsleistung einhergeht, das wird sich als eine ausschlaggebende Verbindungslinie zwischen dem Eposdiskurs um 1800 und demjenigen der klassischen Moderne erweisen. ${ }^{39}$

Die seit 175 o beobachteten und von der neueren sozial- und geschichtswissenschaftlichen Forschung einhellig als Symptomatik der Modernisierung beschriebenen ${ }^{40}$ Phänomene von Bewegung und Beschleunigung manifestieren sich an der Epochenschwelle als konkrete sowie radikale Akzeleration der soziopolitischen, kulturellen und medialen Prozesse. Genau darauf verweist Goethe, wenn er das "fürchterliche Zusammenbrechen aller Verhältnisse $^{41}$ im Kern auf eine problematische Beschleunigung zurückführt: Die immer schnellere Abfolge der Ereignisse entleert die Geschichte, reißt das Gewebe der Kultur auseinander und macht all ihre bisherigen Ordnungsysteme und -kategorien fragwürdig. Liest man Goethes Zielsetzung der Italien-Reise - die Beruhigung seines aufgewühlten Gemüts -, seinen auf Kontinuität ausgerichten Kulturbegriff des ,beides zugleich' und den Rekurs auf die gemeinschaftskonstituierende mediale Qualität des mündlichen Erzählens zusammen mit der Rückbesinnung auf das antike Epos, lassen sich zwei ineinandergreifende Beobachtungen machen. Zum einen wird hier dezidiert die Erfahrung einer neuen Zeitlichkeit zum Ausdruck gebracht, die sich primär durch Unberechenbarkeit und Kontingenz auszeichnet. Die kontinuierliche, kollektive Entwicklung aus einer bekannten, verbindlichen Vergangenheit hin $\mathrm{zu}$ einer berechenbaren, gemeinsamen Zukunft löst

39 Vgl. Benjamin, Walter: Das Kunstwerk im Zeitalter seiner technischen Reproduzierbarkeit. In: Ders.: Das Kunstwerk im Zeitalter seiner technischen Reproduzierbarkeit. Drei Studien zur Kunstsoziologie. Frankfurt a. M.: Suhrkamp 1963. S. 7-44 und ders.: Der Erzähler. Betrachtungen zum Werk Nikolai Lesskows. In: Ders.: Gesammelte Schriften, hrsg. v. Hermann Schweppenhäuser und Rolf Tiedemann, Bd. II. Frankfurt a. M.: Suhrkamp 1977. S. 438-465.

40 Vgl. Koselleck, Reinhart: Das achtzehnte Jahrhundert als Beginn der Neuzeit. S. 269-282, hier S. 278 f; Luhmann, Niklas: Systemtheorie der Gesellschaft, hrsg. v. Johannes F. K. Schmidt und André Kieserling, unter Mitarbeit von Christoph Gesigora. Berlin: Suhrkamp 2017 und Giddens, Anthony: The Consequences of Modernity. Cambridge: Polity Press 199o.

41 MA 14. S. 22. 
sich auf - weder die Zeitdimensionen noch die ihnen eigenen kulturellen Strukturen und sozialen Signaturen können nunmehr in einen umfassenden Zusammenhang gebracht werden. „Die drei Dimensionen der Zeit schienen auseinandergebrochen. Die Gegenwart sei zu schnell und zu provisorisch“, heißt es bei Koselleck. ${ }^{42}$ Insofern wird Goethe schon früh auf die Kehrseiten des linearen Zeitpfeils und des ihm eingeschriebenen Glücksversprechens des Fortschritts ${ }^{43}$ aufmerksam. Weiter setzt er der diagnostizierten und kritisierten Beschleunigung bewusst alternative Zeitvorstellungen von Langsamkeit, Kontinuität und Gleichzeitigkeit entgegen. Es sind gerade solche Elemente der Beharrung, die Hartmut Rosa in seiner vielbeachteten Studie zur Zeitstruktur der Moderne als bewusst widerständige Reaktionen auf die problematische Erfahrung beschleunigter Zeit beschreibt und sie dadurch als inhärente und

42 Koselleck, Reinhart: Vergangene Zukunft. Zur Semantik geschichtlicher Zeiten. Frankfurt a. M.: Suhrkamp 1979. S. 199.

43 Vgl. hierzu Rosa, Hartmut: Beschleunigung. Die Veränderung der Zeitstrukturen in der Moderne (= Suhrkamp Taschenbuch Wissenschaft 1760). Frankfurt a. M.: Suhrkamp 2005. S. 279-294: Das moderne Zeitbewusstsein ist linear mit offenem Ende, unbestimmter Zukunft - es droht kein apokalyptisches Weltende, der Mensch hat ,unendlich` viel Zeit und die Zukunft kann selbst bestimmt werden. Als Verheißung der Beschleunigung dient etwa das Versprechen einer immerwährenden Prosperität, also unbegrenztes Wachstum. Wer schneller leben kann, hat mehr vom Leben bzw. kann sich mehr Erfahrungs- und Erlebnismöglichkeiten erschließen. Das ist eine kapitalistische Glücksökonomie. Deren Versprechen verkehren sich aber auch in Erwartungen und Zwänge (es muss immer schneller immer mehr produziert werden - man muss so viel wie möglich erleben, um das eigene Leben als wertvoll wahrzunehmen) und auch in die Ängste, abgehängt zu werden, den Anschluss zu verpassen, nicht genug aus der Lebenszeit herausgeholt zu haben. In den immer schnelleren Wechseln wird es immer schwieriger, Sinn zu erkennen; verhindert wird auch das Gefühl, am Ende des Lebens, gesättigt' oder weise und gereift zu sein, denn alles, was erlebt, erreicht und an Einsichten gewonnen wurde, ist längst überholt, irrelevant und ungültig geworden. Daraus entwickelt sich eine düstere Perspektivlosigkeit, Lethargie und Gleichgültigkeit: Die Zukunft ist ungewiss, der Einzelne auf sich selbst zurückgeworfen, allein in einer unübersichtlichen, enteilenden Welt, in der nichts bleibt und Bestand hat - die absolute Freiheit führt in Ratlosigkeit, Überforderung und Erstarrung. Entschleunigung avanciert so zum Glücksversprechen der Spätmoderne bzw. der Gegenwart. Glücksökonomie ist heute ein prekärer Balance-Akt zwischen gewünschter Beschleunigung in bestimmten Feldern und zu bestimmten Zeitpunkten (z.B. Kommunikationstechnologie, beim Skype-Anruf wird maximale Geschwindigkeit der Datenübertragung selbstverständlich erwartet) und bewusster Entschleunigung in anderen Feldern zu anderen Zeitpunkten (kontemplative Freizeitgestaltungen wie beispielsweise Yoga-Retreats in Klostern). Vgl. dazu auch Lübbe, Hermann (Hrsg.): Zeit-Erfahrungen. Sieben Begriffe zur Beschreibung moderner Zivilisationsdynamik (= Abhandlungen der Geistes- und Sozialwissenschaftlichen Klasse, Akademie der Wissenschaften und der Literatur, Nr. 5). Stuttgart: Steiner 1996. 
konstitutive Elemente des Moderneprozesses ausweist. ${ }^{44}$ Es ist deshalb eine Hauptthese dieser Arbeit, dass Goethes intensive Beschäftigung mit dem Epischen der 179oer Jahre nicht unbedingt als ein konservativer Abwehrreflex auf die politischen Erschütterungen der Revolution gelesen werden muss, ${ }^{45}$ sondern darin vielmehr eine frühe Einsicht in die viel grundlegendere und irreversible Destabilisierung der Temporalstrukturen sichtbar wird. Bereits die erste, unorthodoxe Auseinandersetzung mit der Gattung ${ }^{46}$ führt Goethe zur Erkenntnis, dass keine epischen Texte mehr geschrieben werden können, ohne die historische Gegenwart zu reflektieren. Daraus erhält er entscheidende Anregungen dazu, wie das Epische an das neue Zeitregime der Moderne angeschlossen werden kann bzw. sogar muss.

\subsubsection{Goethes Morphologie}

Von besonderer Bedeutung sind in diesem Zusammenhang auch die Überlegungen zur Morphologie, in denen Goethe die zuvor vielleicht eher noch diffuse Beunruhigungsbekundung angesichts der medialen Beschleunigungsprozesse im Umfeld der Revolution zu einer konkreten Zeitlichkeitsdiagnose zuspitzt. So richtet sich der Versuch, die Metamorphose der Pflanzen zu klären von 179 o etwa nicht darauf, eine vollendete Gestalt zu beschreiben, sondern vielmehr auf das Sichtbarmachen der organisch-natürlichen Transformationsprozesse, die Veränderungen der Gestalt über die Zeit: „Ein jeder, der nur das Wachsthum [sic] der Pflanzen einigermaßen beobachtet, wird leicht bemerken, dass gewisse äußere Theile [sic] derselben, sich manchmal verwandeln und in die Gestalt der nächstliegenden Theile bald ganz, bald mehr oder weniger übergehen. ${ }^{47}$ Diese "geheime Verwandtschaft der verschiedenen äußern Pflanzentheile [sic]“, „ die Wirkung, wodurch ein und dasselbe Organ sich uns mannigfaltig verändert sehen lässt, [wird] die Metamorphose der Pflanzen genannt ${ }^{448}$, konstatiert Goethe und verschränkt damit das Bewegliche positiv mit dem Lebendigen.

Die Natur ist in dieser Vorstellung ein dynamisches Gestaltkontinuum, in dem jede Form mit jeder anderen verbunden ist, und zwar durch eine

\footnotetext{
44 Dazu, inwiefern der Beginn Moderne von einer umfassenden Beschleunigungserfahrung geprägt ist, vgl. Rosa: Beschleunigung. Die Veränderung der Zeitstrukturen in der Moderne. S. 46o-466 und Koselleck: Das achtzehnte Jahrhundert als Beginn der Neuzeit. S. $269-282$, hier S. 278 .

45 Vgl. MA 4.1. S. 920-922.

46 Vgl. S. 55-74 in diesem Buch.

47 MA 3.2. S. $319 \mathrm{f}$.

48 HA 13. S. 64.
} 
beliebig lange Reihe von Zwischenformen. ${ }^{49}$ Die Morphologie beschäftigt sich also primär damit, die Verhältnisse der Pflanzenwelt in ihrer lebendigen Mannigfaltigkeit - in ihren Verwandlungen, ihrer inneren Organisation und ihrer Beziehung zu anderen - zu erfassen. Dabei geht es Goethe dezidiert darum, in der unendlichen Formenvielfalt eine Regelhaftigkeit der "geheimen Verwandschaft“ und eine Organisationsstruktur in der "mannigfachen Veränderung" sichtbar zu machen, die aus den Teilen ein Ganzes bilden. Er richtet seine Aufmerksamkeit also schon früh auf die Übergänge zwischen den Formen und somit auf die generative Leistung der Form selbst. Diesen im Kern auf ein perpetuierendes Bewegungsmoment ausgerichteten Gestaltbegriff wird Goethe dann in der 2. Auflage der Morphologie von 1817 deutlich zu einem zeitlich bestimmten präzisieren:

Der Deutsche hat für den Komplex des Daseins eines wirklichen Wesens das Wort Gestalt. Er abstrahiert bei diesem Ausdruck von dem Beweglichen, er nimmt an, dass ein Zusammengehöriges festgestellt, abgeschlossen und in seinem Charakter fixiert sei. Betrachten wir aber alle Gestalten, besonders die organischen, so Enden wir, dass nirgend ein Bestehendes, nirgend ein Ruhendes, ein Abgeschlossenes vorkommt, sondern dass vielmehr alles in einer steten Bewegung schwanke. Daher unsere Sprache das Wort Bildung sowohl von dem Hervorgebrachten, als von dem Hervorgebrachtwerdenden gehörig genug zu brauchen pflegt. Wollen wir also eine Morphologie einleiten, so dürfen wir nicht von Gestalt sprechen; sondern, wenn wir das Wort brauchen, uns allenfalls dabei nur die Idee, den Begriff oder ein in der Erfahrung nur für den Augenblick Festgehaltenes denken. [Hervorhebung M.E. $]^{50}$

Goethe artikuliert hier einerseits, was von der Kategorie der Gestalt ${ }^{51}$ erwartet oder vielmehr erhofft wird - nämlich Eindeutigkeit, Abgeschlossenheit und Stabilität - und andererseits hält er gerade auch fest, dass diese Erwartungen angesichts der sich sowohl in der Natur wie eben auch im Kunstsystem abzeichnenden Kräfte der Vervielfältigung und der Dynamisierung nicht aufrechterhalten lassen. Anstatt auf der Gültigkeit von starren Klassifikationen

49 Vgl. Geulen, Eva: Aus dem Leben der Form. Goethes Morphologie der Nager. Köln: August 2016 und Maatsch, Jonas: Morphologie und Moderne. Einleitung. In: Ders. (Hrsg.): Morphologie und Moderne. Goethes „anschauliches Denken“ in den Geistes- und Kulturwissenschaften seit 1800 (= Klassik und Moderne, Bd. 5). Berlin, Boston: de Gruyter 2014. S. 1-15, hier S. 2.

5 O HA 13. S. $54 \mathrm{f}$.

$5^{1}$ Zum naturwissenschaftlichen Gestaltbegriff vgl. den grundlegenden Aufsatz von Kuhn, Dorothea: Grundzüge der Goetheschen Morphologie. In: Goethe Jahrbuch 95 (1978). S. 199-211. Zur "Gestalt“ als ästhetischer Kategorie vgl. Simonis, Annette: „Gestalt" als ästhetische Kategorie. Transformationen eines Konzepts vom 18. bis 20. Jahrhundert. In: Maatsch (Hrsg.): Morphologie und Moderne. S. 245-265. 
zu beharren, beruht Goethes morphologischer Formbegriff auf der Einsicht einer sich nur flüchtig manifestierenden Gestalt und macht entscheidende Zugeständnisse an ein universales, auf Wandelbarkeit und Mannigfaltigkeit $^{52}$ beruhendes Lebensprinzip. Gleichzeitig versucht er aber auch, an der Idee einer strukturierenden, einheitsstiftenden Form festzuhalten. In seinen späteren Naturbetrachtungen konzentriert sich Goethe darauf, „die mannigfaltigen, besondern Erscheinungen des herrlichen Weltgartens auf ein allgemeines, einfaches Prinzip zurückzuführen."53 Denn obwohl Goethe den spontanen, generativen Trieb der Natur und seine Dynamik als übergreifendes produktives Grundprinzip schätzt, misstraut er dennoch dessen zügelloser, chaotischer Kraft:

Die Idee der Metamorphose ist eine höchst ehrwürdige, aber zugleich höchst gefährliche Gabe von oben. Sie führt ins Formlose, zerstört das Wissen, löst es auf. Sie ist gleich der vis centrifuga und würde sich ins Unendliche verlieren, wäre ihr nicht ein Gegengewicht zugegeben: ich meine den Spezifikationstrieb, das zähe Beharrlichkeitsvermögen dessen was einmal zur Wirklichkeit gekommen. Eine vis centripeta, welcher in ihrem tiefsten Grunde keine Äußerlichkeit etwas anhaben kann. [Hervorhebung M.E.] ${ }^{54}$

Die Gesetze der Metamorphose werden hier als zwei entgegengesetzte Kräfte der vis centrifuga und der vis centripeta bezeichnet, wobei die vis centrifuga das transformative Moment bildet, welches in die Unendlichkeit führt und Form und Wissen auflöst. ${ }^{55} \mathrm{Ihr}$ Gegengewicht ist die beharrende Kraft der vis centripeta, die auf eine wenn auch nur temporär verfestigte Form führt.

An der hier formulierten Problematik von Fassbarkeit und Auflösung, die letztlich auch die von Freiheit und Begrenzung ist, arbeitet sich um 1800 das ganze literarische Feld in vielfältiger Weise ab. Eine Problematik, die - wie bereits angedeutet - eng verknüpft ist mit der Dynamisierung und Verzeitlichung der Erfahrungswelt. Die frühromantische Poetik nach Johann Fichte und Friedrich Schlegel greift diese Phänomene nicht nur dankbar auf, sondern installiert sie als geradezu unhinterfragbare, absolute Maximen der neuen, autonomen Ästhetik. ${ }^{56}$ In für diese Anspruchshaltung bezeichnender

\footnotetext{
$5^{2}$ Vgl. Geulen: Aus dem Leben der Form. Goethes Morphologie der Nager. S. 17 f.

53 HA 13. S. 102.

54 MA 12. S. 295.

55 Vgl. Keller: Lebendiger Abglanz. Goethes Italien-Projekt als Kulturanalyse. S. 73 f.

56 Zur Bedeutung des Bewegungsbegriffs für die Frühromantik vgl. Oschmann, Dirk: Zwischen Ästhetik und Mechanik. Frühromantische Visionen der Dynamisierung. In: Ehrlich, Lothar; Schmidt, Georg (Hrsg.): Ereignis Weimar-Jena. Gesellschaft und Kultur um 180o im internationalen Kontext. Wien, Köln, Weimar: Böhlau 2008. S. 129-143.
} 
rhetorischer Formelhaftigkeit und Selbstverständlichkeit fasst Fichte die "Bewegung" in seiner Practischen Philosophie als "bekanntlich [...] unendlicher Begriff “ ${ }^{57}$ Wie Oschmann darlegt, schließt Fichte hier an die Eroberung des Bewegungsdiskurses durch den englischen Empirismus an, gründet seinen unendlichen Bewegungsbegriff aber anders als etwa der Sensualismus Moritzens auf die physikalische Mechanik. ${ }^{58}$ Damit verknüpft er aber nicht nur modernistisch Bewegung und Unendlichkeit, sondern denkt die Verbindung auch mit dem Begriff der Freiheit zusammen: „Bewegung ist auch wirklich das erste, wodurch die Natur [Prise] über uns giebt [sic]. [...] Es ist der erste [Begriff], der Leben, ein Analogon von Freiheit in die Natur bringt.“59

Einen ähnlich positiven Fokus auf das entgrenzende, freisetzende Moment der Bewegung legen auch die Brüder Schlegel, die das Bewegungsparadigma aber stärker poetologisch-ästhetisch funktionalisieren: Die Poesie tritt August W. Schlegel zufolge „überall und zu allen Zeiten in irgend einer gemessenen Bewegung auf "60 und sein Bruder Friedrich definiert seinen zentralen philosophischen Ideen-Begriff als „unendliche, selbständige, immer in sich bewegliche, göttliche Gedanken". ${ }^{61}$ Für das Selbstverständnis einer fortschrittsgläubigen Moderne ist diese frühromantische Engführung von Bewegung, Freiheit und Unendlichkeit geradezu konstitutiv - darin manifestiert sich nämlich das bis heute wirksame Glücksversprechen einer unendlichen Beschleunigung und Mobilität. ${ }^{62}$ Das Unspezifische und Formlose imaginieren die Frühromantiker als positive Effekte einer stets vorwärtsschreitenden Zeit, in der die Gegenwart als flüchtiger, futuristisch geprägter Moment erscheint. Damit wenden sie sich vom Zeithorizont der Vergangenheit

57 Fichte, Johann Gottlieb: Practische Philosophie (1794). In: Ders.: Gesamtausgabe der Bayrischen Akademie der Wissenschaften, hrsg. v. Lauth, Reinhart; Jacob, Hans, Bd II/3, Nachgelassene Schriften 1793-1795. Stuttgart, Bad Cannstatt: Frommann 1971. S. 179-266, hier S. 243 .

$5^{8}$ Vgl. Oschmann, Dirk: Zwischen Ästhetik und Mechanik. Frühromantische Visionen der Dynamisierung. S. 129-143, hier S. 131-133.

59 Fichte: Practische Philosophie (1794). S. 179-266, hier S. 243 f.

6o Schlegel, August Wilhelm: Briefe über Poesie, Silbenmaß und Sprache. In: Ders.: Sämtliche Werke, hrsg. v. Eduard Böcking, Bd VII/1. Vermischtes und kritische Schriften. New York, Hildesheim: Georg Olms Verlag 1971. S. 98-154, hier S. 108.

61 Schlegel, Friedrich: Ideen (Erstes und Zweites Stück, 180o). In: Athenaeum. Eine Zeitschrift, hrsg. v. August Wilhelm Schlegel u. Friedrich Schlegel; mit einem Nachwort von Ernst Behler (1992). S. 4-33, hier S. 5 .

62 Vgl. Oschmann: Zwischen Ästhetik und Mechanik. Frühromantische Visionen der Dynamisierung. S. 129-143, hier S. 135. Die zukunftseuphorische Verknüpfung von Beschleunigung und Moderne und inwiefern diese auch Probleme birgt, wird im Folgenden noch ausführlicher besprochen. 
entschieden ab und halten sich dagegen an das Jetzt als Exponent einer zeitlichen Wende hin auf die Zukunft. ${ }^{63}$

Von dieser Emphase der Aufbruchsdisposition unterscheidet sich nun aber die Verknüpfung von Bewegung und Zeitlichkeit in Goethes morphologischer Formenlehre deutlich. Die zeitliche Bewegung des Lebens nimmt darin zwar die zentrale Stelle ein, wird aber bei weitem nicht so radikal freigesetzt wie in der frühromantischen Literaturtheorie. Vielmehr wird die Vorwärtsbewegung der Zeit an ein zähes Beharrlichungsvermögen gebunden, das die bei Goethe gefährliche Auflösung ins Unendliche gerade verhindert und dadurch an der Vorstellung von Kontinuität festhält. Damit richtet Goethe die Morphologie nicht nur auf die Gestaltwerdung, sondern auch wesentlich auf die Möglichkeit aus, die fortschreitende Zeit zu verdichten: So wie im Blatt der Pflanze gleichwohl die frühere Wurzel als auch die späteren Blüten ersichtlich und damit fassbar werden, so wirkt im gegenwärtigen, durch die vis centrifuga festgehaltenen Augenblick noch die vorhergegangene Vergangenheit nach und die kommende Zukunft kann bereits erahnt werden.

\subsubsection{Prägnanz}

Die Fixierung des flüchtigen Moments in einer Gleichzeitigkeit von Vergangenheit, Gegenwart und Zukunft steht nicht nur als Zeitlichkeitskonzeption dem romantischen Jetzt diametral entgegen, sondern bedeutet vor allem auch einen bewussten Paradigmenwechsel in Goethes Formdenken hin zu einer vorrangig zeitlich verfassten Ästhetik: „Das Hauptanliegen von Goethes Formbegriff ist“, wie Sabine Schneider ausführt, „die Erfahrbarkeit gegliederter, prägnanter Sequentialität, erfüllter Gegenwart und kontinuierlicher Abfolge, das Bewohnen der Vergangenheit und der Zukunft in der erlebten Gegenwärtigkeit." ${ }^{44}$ Diese gleichwohl ambivalenten wie ambitiösen Ansprüche an die Form lassen sich im Begriff der Prägnanz zusammenfassen: Prägnant ist die Form, gemäß Schneider, wenn sie maximale Bedeutungsfülle mit minimalem Darstellungsaufwand erzeugt, oder: „das Endliche geht schwanger mit dem Unendlichen.“65 „Keine Zeit und keine Macht“, heißt es dann bei Goethe

63 Vgl. Oesterle, Ingrid: „Es ist an der Zeit!“ Zur kulturellen Konstruktionsveränderung von Zeit gegen 180o. In: Walter Hinderer, Alexander von Bormann, Gerhart von Graevenitz (Hrsg.): Goethe und das Zeitalter der Romantik (= Stiftung für Romantikforschung, Bd. 21). Würzburg: Königshausen \& Neumann 2002. S. 91-121, hier S. 102 f.

64 Schneider, Sabine: „ein strenger Umriß“ - Prägnanz als Leitidee von Goethes Formdenken im Kontext der Weimarer Kunsttheorie. In: Goethe Jahrbuch 128 (2011). S. 98-106, hier S. 100 .

65 Ebd. S. 99: Prägnanz von lat praegnans: schwanger. 
selbst, „zerstückelt/Geprägte Form, die lebend sich entwickelt““66 Im Wesentlichen leistet also die prägnante Form, im Gegensatz zur formlosen Form, wie sie etwa Friedrich Schlegel imaginieren wird, ${ }^{67}$ eine ästhetische Konzentration oder Verdichtung, ${ }^{68}$ so dass überhaupt erst fassbar wird - wenn auch nur für einen Moment - was sich ansonsten als unstrukturierte, instabile und grenzlose Masse präsentiert.

Mit der Idee der Prägnanz schließt Goethe gewissermaßen an Lessings medienpoetologische Ausdifferenzierung der Künste in der LaokoonAbhandlung an. Aus dem scharfen Verweisen der Bildkunst auf das Feld der räumlichen Wirkungsdimension auf der einen Seite und der Literatur auf das Feld der zeitlichen auf der anderen ergab sich hier für die bildende Kunst das Problem der Zeitbehandlung69 sowie für die narrative Kunst das Problem der Raumbehandlung. Im Falle der Bildkunst versucht Lessing das Problem durch die Vorstellung eines „prägnanten Moments“ zu lösen, in dem das „Vorhergehende und Folgende am begreiflichsten ${ }^{470}$ gefasst werden können. Die Herausforderung für die bildende Kunst besteht also darin, denjenigen Augenblick einer Handlungsabfolge darzustellen, der es dem Betrachter ermöglicht, die vorausgehenden und nachfolgenden Momente der Handlung aus der eigenen Imagination zu konstituieren. Damit erweist sich Lessings Denken bezeichnendereweise auch in Bezug auf die darstellende, räumlich organisierte Kunst von narrativen bzw. zeitlichen Kategorien geprägt. ${ }^{71}$

Wenn Goethe seinserseits die Idee der Prägnanz nun allerdings für ein kunstübergreifendes, allgemeines Formdenken produktiv macht, das sich dezidiert gegen das Sukzessivitätsgebot der Zeit stellt, überbietet er damit

66 Goethe, Johann Wolfgang: Urworte, orphisch In: Gesammelte Werke in sieben Bänden, hrsg. v. Bernt von Heiseler, Bd. 1. Gütersloh: Bertelsmann 1954. S. 403.

67 Vgl. Wohlleben: Die Sonne Homers. Zehn Kapitel deutscher Homer-Begeisterung. Von Winckelmann bis Schliemann. S. 62 und Matuschek, Stefan: „Doch Homeride zu sein, auch nur als letzer, ist schön“. Zur Bedeutung der Mythologie bei Friedrich Schlegel. S. 123.

68 Vgl. Oesterle: „Es ist an der Zeit!“ Zur kulturellen Konstruktionsveränderung von Zeit gegen 180o. S. 91-121, hier S. 104.

69 Vgl. Schneider, Sabine: Die Laokoon-Debatte: Kunstreflexion und Medienkonkurrenz im 18. Jahrhundert. In: Benthien, Claudia; Weingart, Brigitte (Hrsg.): Handbuch Literatur und Visuelle Kunst. Berlin, Boston: de Gruyter 2014. S. 68-85, hier S. 79.

70 Lessing, Gotthold Ephraim: Laokoon. Sämtliche Schriften, hrsg. v. Karl Lachmann, Bd. 9. Leipzig, Stuttgart: Göschen 1893. S. 19 u. 95.

71 Zur Interferenz zwischen Lessings und Goethes Prägnanz-Begriffen vgl. auch Wolf, Norbert Christian: „Fruchtbarer Augenblick“ - „prägnanter Moment“: Zur medienspezifischen Funktion einer ästhetischen Kategorie in Aufklärung und Klassik (Lessing, Goethe). In: Alt, Peter-André; Košenina, Alexander et al. (Hrsg.): Prägnanter Moment. Studien zur deutschen Literatur der Aufklärung und Klassik. Festschrift für Hans-Jürgen Schings. Würzburg: Königshausen \& Neumann 2002. S. 373-404. 
Lessings Medienästhetik ganz entschieden. Darüber hinaus wirft er vor allem auch einen kritischen Blick auf deren Gegenstandslehre, ${ }^{72}$ in dem er die spezifische zeitliche Verfasstheit des Bildhaften - die ästhetische Gleichzeitigkeit für die Literatur, besonders für das Erzählen fruchtbar macht. Explizit taucht der Prägnanz-Begriff erst in Goethes Spätwerk bzw. im Nachlass auf ${ }^{73}$ und erlangt hier den Status einer Leitidee. Im Grunde handelt es sich dabei allerdings um den gleichen Gedanken, wie er bereits in der Morphologie artikuliert wird, nämlich um das Anliegen, die wohl produktiven, aber auch anarchischen Prinzipien des Lebendigen - Mannigfaltigkeit und Beweglichkeit $^{74}$ - in der Zeit zu bannen. Wichtige Anregungen dazu hatte Goethe schon während der Italien-Reise erhalten, wie der Brief an Herder aus dem Jahr 1787 offenbart, in dem Goethe die vielfältige und wechselvolle Erscheinungswelt Italiens „im Geiste gegenwärtig ${ }^{475}$ halten will. Insofern fühlt Goethe die Paradigmen und Problemstellungen, die im spätwerklichen Begriff der Prägnanz enthalten sein werden, sowohl in der kulturanalytischen Betrachtung Italiens wie auch in der Morphologie der Pflanzen maßgeblich vor, indem er in beiden Bereichen auf einer Kontinuitätslinie zwischen dem Vergangenen, dem Gegenwärtigen und dem Zukünftigen beharrt. Wie es im Weiteren noch aufzuzeigen gilt, steht das Aufmerksamwerden auf die Möglichkeiten und Gefahren der Verzeitlichung auch in direkter Verbindung mit Goethes Epos-Projekt und den darin aufgeworfenen Fragen nach einem neuen adäquaten Formbegriff. Die Auseinandersetzung mit dem epischen Erzählen wird sich als wichtige Inkubationszeit erweisen, in der die spätere Denkfigur der Prägnanz diskursiv erarbeitet und ausgestellt wird.

72 Vgl. Schneider: Die Laokoon-Debatte: Kunstreflexion und Medienkonkurrenz im 18. Jahrhundert. S. 68-85.

73 Wie etwa die folgende Stelle: „Alles Prägnante, was allein an einem Kunstwerk vortrefflich ist, wird nicht erkannt, alles Fruchtbare und Fördernde wird beseitigt, eine tiefumfassende Synthesis begreift nicht leicht jemand.“ Zitiert nach Schneider: „ein strenger Umriß“ - Prägnanz als Leitidee von Goethes Formdenken im Kontext der Weimarer Kunsttheorie. S. 98.

74 Vgl. Geulen: Aus dem Leben der Form. Goethes Morphologie der Nager. S. 17 f.

75 Vgl. S. 85 in diesem Buch. 


\subsection{Goethes explorative Epistemologie als Gegenmodell zum Empirismus}

Als früher Hinweis auf die für Goethe je länger desto charakteristischere Synthese von kunsttheoretischem und naturwissenschaftlichem Formdenken, ${ }^{76}$ von Ästhetik und Wissenschaft zu einem Wissenszusammenhang, kann zunächst die Beschreibung einer in Italien bereits intuitiv betriebenen, explorativen Forschungsmethode dienen:

Im Laufe von zwei vergangenen Jahren hatte ich ununterbrochen beobachtet, gesammelt, gedacht, jede meiner Anlagen auszubilden gesucht. [...] so dass ich hoffen konnte nach und nach das Ganze zu überschauen, und mir einen reinen, vorurteilsfreien Kunstgenuss zu bereiten. Ferner glaubte ich der Natur abgemerkt zu haben, wie sie gesetzlich zu Werke gehe, um lebendiges Gebild, als Muster alles künstlichen, hervorzubringen. ${ }^{77}$

Beobachtung und Sammlung, die sukkzessive Annäherung an ein Ganzes über seine Teile und die Überzeugung von der prozesshaften Verfasstheit allen Lebens zeichnen sich hier am Gegenstand der Kunstbetrachtung bereits als die ausschlaggebenden Begriffe einer alternativen erkenntnisorientierten Tätigkeit ab. Diese entwickelt Goethe dann im Zusammenhang mit seiner naturwissenschaftlichen Beschäftigung zu einer Methodik weiter, die der integralen Verwandtschaft und Vielfalt aller lebensweltlichen Erscheinungen gerecht werden soll. ${ }^{78}$

\subsubsection{Offener Versuch anstatt experimentum crucis}

Der Versuch als Vermittler von Objekt und Subjekt von 1792 befasst sich deshalb eingehend mit dem Experiment als neuer wissenschaftlicher Schlüsselkategorie und greift dabei grundsätzliche Problemstellungen der zeitgenössischen Naturwissenschaft auf. Darin bezieht Goethe kritisch Stellung gegen Newtons positivistische Forschungsmethode ${ }^{79}$ und entwickelt gleichzeitig sein eigenes

76 „Hier sah ich meine disparatesten Beschäftigungen neben einander gestellt, Kunst- und Naturerzeugnisse eins behandelt wie das andere, ästhetische und teleologische Urteilskraft erleuchteten sich wechselseitig." FA I, 24. S. 444.

77 HA 13. S. 101.

78 Vgl. Geulen, Eva: Keeping it Simple, Making it Difficult: Morphologische Reihen bei Goethe und anderen. In: Koschorke, Albrecht (Hrsg.): Komplexität und Einfachheit: DFG-Symposium 2015. Stuttgart: Metzler 2017. S. 357-373, hier S. 36o.

79 Vgl. MA 4.2. S. 268. Zu Goethes Auseinandersetzung mit Newton vgl. Cassirer, Ernst (Hrsg.): Goethe und die mathematische Physik. Eine erkenntniskritische Betrachtung. In: Idee und Gestalt: Goethe, Schiller, Hölderlin, Kleist. Darmstadt: Wissenschaftliche 
Verständnis des Experimentierens als enge Verflechtung von erkenntnistheoretischer Reflexion und methodologischer Innovation. ${ }^{80}$

Die eigentliche Kernfrage, um die Goethes Versuch-Aufsatz kreist, ist die nach der Möglichkeit einer vorurteilsfreien, objektiven Beobachtung in der theoretischen Wissenschaft. ${ }^{81}$ Die Epistemologie der Forschung, die er daraus entwicklet, seine Wahrnehmung des Verhältnisses der einzelnen Teile zum allgemeinen Ganzen sowie der Beziehungen zwischen Objekt und Subjekt, dem Betrachter und dem Betrachteten, kommt einer alternativen Wissenschaftlichkeit gleich. Dazu gehört etwa die Vorstellung einer interagierenden wissenschaftlichen Gemeinschaft, die sich über das gemeinsame Interesse von Forschenden konstituiert. Goethe schreibt von einer Methode, „mit Mehreren zu arbeiten“, mit der er selbst bereits gute Erfahrung gemacht hat und die darin besteht, „an[zu]erkennen, wie nötig Mitteilung, Beihülfe, Erinnerung und Widerspruch sei, um uns auf dem rechten Weg zu erhalten und vorwärts zu bringen“. ${ }^{82}$ In naturwissenschaftlichen Belangen ist es deshalb, ganz anders als in der Kunst, nicht nur möglich sondern geradezu erforderlich, ,jede einzelne Erfahrung, wider Vermutung öffentlich mitzuteilen, ja es ist höchst rätlich, ein wissenschaftliches Gebäude nicht eher aufzuführen, bis der Plan dazu und die Materialen allgemein bekannt, beurteilt und ausgewählt sind“ ${ }^{483}$ In der Forschergemeinschaft können nach Goethe also Erfahrungen gewonnen und gesammelt werden und so zu einer objektiven, gültigen Beschreibung des Gegenstands führen. Während der Künstler wohl tut, „sein Kunstwerk nicht öffentlich sehen zu lassen, bis er es vollendet hat", ist es für den Forscher mehr als legitim, mit einem unfertigen Ergebnis, einem Versuch in die Öffentlichkeit zu treten. ${ }^{84}$

Buchgesellschaft 1971. S. 33-80 und Steinle, Friedrich: „Das Nächste ans Nächste reihen“: Goethe, Newton und das Experiment. In: Philosophia naturalis 1 (2002). S. 141-172.

8o Darin liegt die eigentliche Relevanz seines Versuchs. Anders als in der Naturwissenschaft, in der sich Goethes Ansatz nie durchsetzen konnte, trägt er für die Geistes- und Kulturwissenschaften wesentlich zu einem neuen Wissenschaftsverständnis bei, das auf das Problembewusstsein der Moderne reagiert, vgl. Maatsch: Morphologie und Moderne. Einleitung. S. 1-15, hier S. 10.

81 Vgl. MA 4.2. S. 321-323.

82 Ebd. S. 324 f.

83 Ebd. S. 325 .

84 M.A. 4.2. S. 325. Das Serielle, Fragmentarische, Unfertige ist eine legitime Erscheinungsform für naturwissenschaftliche Arbeiten - wie enthusiastisch Goethe diese Offenheit annimmt, beweisen die zahlreichen Versuche und Beiträge - an Kunstwerke wird aber der Anspruch von Ganzheit, Abgeschlossenheit gestellt. 
Der Kern des gemeinschaftlichen Versuchs, dessen Ziel das Teilen von Erfahrungen ist, steht die Vervielfältigung und die setzt sich bezeichnenderweise über jegliche zeitlichen Einschränkungen hinweg: „Wenn wir die Erfahrungen welche vor uns gemacht worden, die wir selbst oder andere zu gleicher Zeit mit uns machen, vorsätzlich wiederholen und die Phänomene [...] wieder darstellen, so nennen wir dieses einen Versuch. ${ }^{\text {“85 }}$ Dieser Vorstellung ist die Forderung nach Variantenreichtum eingeschrieben, nach einer Differenzierung des Untersuchungsbereichs in möglichst viele experimentelle Phänomene. Freilich ist von dieser Methodologie des Wiederholens und Variierens keine empirisch eindeutige Aussage, sondern vielmehr eine Resultathäufung zu erwarten, die sich stark durch Flächigkeit, Breite und Heterogenität auszeichnet. Für Goethe liegt aber gerade in der Vielfalt das eigentliche Erkenntnispotenzial begründet, denn ,jeder Versuch“ erhält „seinen Wert durch Vereinigung und Verbindung mit anderen." ${ }^{86}$ Je mehr Versuche angestellt werden, desto leichter lassen sie sich „in eine recht natürliche Verbindung" bringen, je mehr Verbindungen zu Tage treten, desto plausibler wird die daraus abgeleitete Erkenntnis: „Eine jede Erfahrung die wir machen, ein jeder Versuch, durch den wir sie wiederholen, ist eigentlich ein isolierter teil [sic] unserer Erkenntnis, durch öftere Wiederholung bringen wir diese isolierte Kenntnis zur Gewissheit." ${ }^{\text {"87 }}$

Die Heterogenität und Partikularität der Erscheinungswelt verlangt für Goethe konsequenterweise auch nach einer Erkenntnisweise, die die Fülle und Gleichzeitigkeit der konkreten sinnlichen Anschauung mit der synthetischen Kraft des verallgemeinernden Verstandes verbindet. Um „der Mannigfaltigkeit des Seins und Werdens und der sich lebendig durchkreuzenden Verhältnisse ${ }^{488}$ gerecht zu werden, stellt Goethe deshalb in seiner Farbenlehre eine große Fülle von Versuchen und Beobachtungen an, die die Umstände und Bedingungen, die Art und Weise von Farberscheinungen in all ihren Facetten kontinuierlich ausbreitet, ohne dabei eine Gewichtung oder Hierarchisierung vorzunehmen.

An diesem Punkt offenbart sich nun auch im methodologischen Denken eine spezifisch zeitliche Implikation: Es geht Goethe nicht darum, so schnell wie möglich vom partikularen Versuch auf ein allgemeines Urteil zu kommen, sondern sich Zeit zu lassen. „Ungeduld, Vorschnelligkeit, Selbstzufriedenheit, Steifheit, Gedankenform, vorgefasste Meinung, Bequemlichkeit, Leichtsinn“

\begin{tabular}{ll}
\hline 85 & Ebd. \\
86 & Ebd. S. 326. \\
87 & Ebd. S. 326 f. \\
88 & HA 13. S. 54.
\end{tabular}


und „Veränderlichkeit“ nennt er als „innere Feinde“, die dem Forscher am Übergang von der Erfahrung zum Urteil zum Verhägnis werden können. ${ }^{89}$ Stattdessen ist mit einer „ruhigen Aufmerksamkeit“ ${ }^{40}$ vorzugehen, denn objektive Zusammenhänge lassen sich erst durch geduldiges Nebeneinanderstellen und Vergleichen allmählich offenlegen. ${ }^{91}$ Das Interesse richtet sich dann auch nicht darauf, von „vielen“ auf „alle" zu schließen, sondern von „Ähnlichem“ auf „Gleiches“ oder "Ungleiches"92 und damit gegen die zeitgenössische Dichotomie der Naturwissenschaften. Goethe sieht in jeder beobachteten „Ähnlichkeit" ${ }^{493}$ zwischen den Ergebnissen sowohl Unterschiede wie auch Gleichheit - bei jeder gefundenen Ähnlichkeit stellt sich damit die Frage, ob sie eher ein Zeichen für einen feinen Unterschied oder für eine annähernde Gleichheit ist. Eine beliebig lange Versuchsreihe über verwandte Phänomene gibt deshalb sowohl über immer weitere Unterschiede wie auch über immer engere Verwandschaft Aufschluss. ${ }^{94} \mathrm{Um}$ die Verbindung zwischen den Phänomenen zu finden, ist es unabdingbar, „alle Seiten und Modifikationen einer einzigen Erfahrung, eines einzelnen Versuchs nach aller Möglichkeit durchzuforschen und durchzuarbeiten ${ }^{495}$. Die Beweise dieser Methode sind Darstellungen davon, „dass dasjenige, was in Verbindung vorgebracht wird, schon in seinen einfachen Teilen und in seiner ganzen Folge da gewesen ${ }^{496}$ ist.

\subsubsection{Dynamisierung und Vervielfältigung}

Die darin liegende Forschungsbewegung ist nicht eine gerade Linie von A nach B, sondern eine kreisende, tastende, explorative. Die Phänomene und ihre Grenzen werden Schritt für Schritt, ohne einen dazwischen auszulassen, ab- und auch überschritten. Das Element der Bewegung wird dadurch ausschlaggebend für die ganze Versuchsanlage, wird der statische Kontext von Hypothese und Bestätigung doch durch den dynamischen Kontext einer Erfahrungserweiterung durch zahlreiche Versuche ersetzt. ${ }^{97}$ Für Goethe ist

$89 \quad$ MA 4.2. S. 326 .

$90 \quad$ MA 4.2. S. 322.

91 Bereits die anatomischen Studien zum Zwischenkieferknochen von 1784 hatten Goethe auf die Spur einer vergleichenden Wissenschaft geführt, inspirierten ihn diese Studien doch zu einem Ersten Entwurf einer Einleitung in die vergleichende Anatomie, den er zwar schon 1795 niederschrieb, aber erst 1820 veröffentlichte. Es blieb jedoch bei der Einleitung, das Projekt führte er nie aus, vgl. MA 4.2. S. 1021.

92 Krohn: Goethes Versuch über den Versuch. S. 399-413, hier S. 408.

93 MA 4.2. S. 326 f.

94 Vgl. Krohn: Goethes Versuch über den Versuch. S. 399-413, hier S. 408.

95 MA 4.2. S. 329 .

96 Ebd. S. 33०.

97 Vgl. Krohn: Goethes Versuch über den Versuch. S. 399-413, hier S. 410. 
Forschen in erster Linie das unvoreingenommene Sammeln von Erfahrungen, neuen und bestehenden. Darin sind einerseits Bewusstein und Wertschätzung für die Wissenstradition und andererseits auch ein Moment des Bewahrens enthalten, im Sammeln können Erfahrungen und Ereignisse der Vergangenheit beibehalten, mehr noch vergegenwärtigt werden. Allerdings geht es dabei nicht darum, das Gewesene museal zu konservieren, sondern vielmehr darum, es auszustellen, sichtbar und präsent zu machen. Auch hier sind also modernes Krisenbewusstsein und Bemühungen, sich gegen den beschleunigten Lauf der Zeit und seiner Auflsösungsmacht aufzulehnen, spürbar. ${ }^{98}$ Denn Sammeln ist auch Arbeit an der Zeit - im Nebeneinanderstellen von alten Erfahrungen und neue Innovationen kann die Wissensgeschichte nämlich nicht zuletzt als Kontinuum beleuchtet werden.

Durch die Untersuchung nicht nur des einen Ereignisses, sondern immer auch dessen, „was unmittelbar an [den Versuch] grenzt ${ }^{499}$, vervielfachen sich die Erfahrungen. „Die Vermannigfaltigung eines jeden einzelnen Versuches

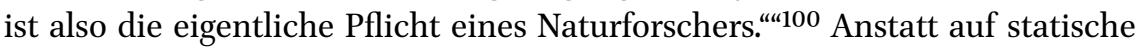
Gegenstände richtet sich das Interesse Goethes eben auf die Bewegung zwischen den Gegenstände und will Prozesse erfassen. Dass ein derartig breites Erkunden besonders davon profitiert, wenn sich viele daran beteiligen und sich darüber austauschen, hat Goethe klar vor Augen. Ihm geht es weder um eine eindeutige, vollständige und abgeschlossene Definition der Forschungsgegenstände, noch um eine einheitliche Betrachtung der Forschungsresultate. Der besondere Gewinn seiner explorativen Epistemologie ist vielmehr, dass die einzelnen Erfahrungen in immer neue Kontexte gestellt, in verschiedenen Reihen, Ketten, Netzen, Konstellationen und Verknüpfungen angeordnet und aus immer wieder anderern Blickwinkeln betrachtet werden können. Der springende Punkt oder das Problem, das sich allerdings hier und eben auch in Bezug auf die Gattung des Epos besonders prominent stellt, ist die Frage nach einer möglichen und sinnvollen Begrenzung: Welche Phänomene werden aus der unüberschaubaren Komplexität der Wirklichkeit aus- bzw. eingegrenzt und wie sollen diese Grenzen etabliert werden? Von der Versuchsanordnung wird schließlich diese Organisationsleistung verlangt:

98 Zum Zusammenhang von Sammlung und Zeit (am Beispiel von Dichtung und Wahrheit) vgl. Böhme, Hartmut: Fetisch und Idol. Die Temporalität von Erinnerungsformen in Goethes Wilhelm Meister, Faust und Der Sammler und die Seinigen. In: Matussek, Peter (Hrsg.): Goethe und die Verzeitlichung der Natur. München: Beck 1998. S. 178-201, hier S. 193 .

99 MA 4.2. S. 329 .

100 Ebd. 


\begin{abstract}
Aber diese Materialien müssen in Reihen geordnet und niedergelegt sein, nicht auf hypothetische Weise zusammengestellt, nicht zu einer systematischen Form verwendet. Es steht alsdenn einem jenen frei, sie nach einer Art zu verbinden und ein Ganzes zu bilden, das der menschlichen Vorstellungart überhaupt mehr oder weniger bequem und angenehm sei. ${ }^{101}$
\end{abstract}

Diese Versuche gilt es dann zu vergleichen und zu ordnen, was wiederum dazu führt, „neue Versuche zu erfinden und die Reihe derselben vollständiger zu machen“.102 Diese Verfahrensweise erzeugt ein geradezu unübersichtliches, changierendes und vor allem unbegrenztes Versuchsfeld, auf dem sich die einzelnen sinnlichen Erfahrungen durch geduldiges Vergleichen in unterschiedliche Zusammenhänge stellen lassen. ${ }^{103}$

In Bezug auf die Reihe als primäre Ordnungsstruktur charakterisiert Joseph Vogl Goethes experimentelle Praxis „weniger an der Reihung von Einzelfällen mit Blick auf Gesetzmäßigkeiten als an einer ,Vermannigfaltigung“ von Versuchen und Erscheinungen ausgerichtet, sie ,vermannigfaltigt' die Farbeffekte bis ins Unendliche und folgt einem de jure unabschließbaren Gang“ ${ }^{104} \mathrm{Er}$ präzisiert damit eine weitere, für diese Arbeit ausschlaggebende Besonderheit in Goethes Formdenken: Die Reihe erscheint zwar als Ordnungsbegriff immer wieder zentral, sowohl in den naturwissenschaftlichen wie auch kunsttheoretischen Betrachtungen, dabei steht für Goethe aber nicht unbedingt die Reihe als innere, konsekutive Struktur eines abgeschlossenen Ganzen im Vordergrund, vielmehr scheint ihm das entgrenzende Potenzial einer kontinuierlichen, unabgeschlossenen Häufung von ahnungsvollen, sinnstiftenden Wiederholungen interessant. In diese Richtung argumentiert auch Geulen, wenn sie konstatiert, dass es in Goethes Auffassung der Reihe ,jenseits des umittelbar Vorausgehenden und Nachfolgenden keinen Bezug [...] zwischen den beiden Elementen der Reihe ${ }^{\text {“105 }}$ gäbe. Goethes Reihe führe weder zu einer These noch zu Totalität, denn Voraussetzung dafür wäre eine vollständige Reihe, die unter den Erkenntnisbedingungen des Menschen aber unmöglich bleiben muss: „Damit ist Reihenbildung kein Verfahren der Beziehungsstiftung, Verkettung, Vernetzung, Verdichtung oder Annäherung ans Ganze, sondern umgekehrt eine weitere Strategie der Bezugsvermeidung, der Verzögerung und des Aufschubs. ${ }^{\text {106 }}$

\footnotetext{
101 MA 4.2. S. 331.

102 MA 4.2. S. 269.

103 Vgl. Vogl, Joseph: Bemerkung über Goethes Empirismus. In: Ders.; Schimma, Sabine (Hrsg.): Versuchsanordnungen 180o. Berlin, Zürich: diaphanes 2009. S. 113-126.

104 Ebd. S. $115 \mathrm{f}$.

105 Geulen: Aus dem Leben der Form. Goethes Morphologie der Nager. S. 116.

106 Ebd.
} 
Darin ist wiederum eine spezifisch modernekritische, zeitliche Verfasstheit der goetheschen Morphologie ersichtlich: Wenn in einer Reihe jeweils nur das unmittelbar vorhergehende und das unmittelbar nachfolgende interessieren, erzeugt das nicht ein lineares Abfolgeverhältnis, sondern kontakttheoretische Gleichzeitigkeit. Goethes Reihe ist, das hat wiederum Eva Geulen durchschaut, gar kein Verfahren zur Beziehungsstiftung, sondern ein Medium der Verzögerung mit Unterbrechungsfunktion. ${ }^{107}$ Insofern bestätigt sich hier, dass Goethe zeitgleich zum Epos-Projekt eine Denkweise entwickelt, die Pluralität und Heterogenität eindeutig positiv bewertet und Wissen als grenzenloses, ungeordnetes Feld begreift. Im Aufsatz denkt Goethe zwar an die Vernetzung vieler eng zusammenhängender Erfahrungen und mehr noch an eine Verflechtung solcher Vernetzungen: Diese künstliche Welt experimentell erzeugter Feinheiten ist die Vermittlungsleistung des Versuchs zwischen der Erfahrungswelt und der geistigen Reflexion. Dass dadurch das Problem der Begrenzung allerdings bei weitem nicht gelöst ist, sondern mehr und mehr in alle Bereiche von Goethes Denken und Schaffen eindringt, beweisen seine lebenslangen Auseinandersetzungen mit der Form - noch 1826 drängt er „immer auf die letzte Formeln hin[...], durch welche ganz allein mir die Welt noch fasslich und erträglich wird“. 108

\subsubsection{Verschränkung von Beobachtung und Gegenstand: Sinnlichkeit als alternativer Erkenntnismodus}

Goethes Programm der Exploration vollzieht sich auf zwei Ebenen: zum einen auf der Ebene des praktischen Arbeitens, des methodischen Vorgehens - des Experimentierens - und zum anderen auf der Ebene der Beobachtung, des Umgangs mit den gewonnen Resultaten - des Erklärens. Und obwohl es sich zwar innerhalb der zeitgenössischen Physik verortete, steht es der damals gültigen Konzeption der Mechanik klar entgegen, lehnt es den ihr zugrundeliegenden cartesianischen Dualismus - die ontologische Prämisse, dass es nur die Ereignisse der Wirklichkeit gibt und deren Wahrnehmung im Bewusstsein doch ab. ${ }^{109}$ Es ging Goethe allerdings kaum darum, sich konfrontativ gegen

\footnotetext{
107 Vgl. Geulen: Keeping it Simple, Making it Difficult: Morphologische Reihen bei Goethe und anderen. S. 357-373, hier S. 363.

108 Brief an Sulpiz Boisserée, WA IV, 41. S. 221, zitiert nach Schneider: „ein strenger Umriß“ Prägnanz als Leitidee von Goethes Formdenken im Kontext der Weimarer Kunsttheorie. S. 98.

109 Goethes Nachdenken über die Tätigkeit des Forschens und die Kommunikation der Forscher wird von den zeitgenössischen wie auch den nachfolgenden Naturwissenschaftlern zurückgewiesen, vgl. Maatsch: Morphologie und Moderne. Einleitung. S. 1-15, hier S. 10. Seine Ablehung des cartesianischen Dualismus wird als grundlegender Irrtum
} 
die damals noch junge experimentalwissenschaftliche Tradition zu stellen. Vielmehr erkannte er wohl hellsichtig die engen Grenzen der cartesianischen Dichotomie, die für alles jenseits der Wirklichkeit und ihrer Beobachtung blind ist, und damit das Dritte dazwischen, das Zugleich von Realität und Reflexion, um das es Goethe geht, kategorisch ausschließt. Goethes Anstrengungen in der Farbenlehre - überhaupt in seinem naturwissenschaftlichen Betätigungen richten sich also auch darauf, den als unzureichend erkannten erkenntnistheoretischen Dualismus zu überwinden und eine Neuorientierung innerhalb der wissenschaftlichen Forschung anzuregen. Gegenüber Johann Friedrich Reichardt äußert er explizit die Hoffnung, seine Beiträge zur Optik möchten „mancherlei Revolutionen sowohl in der Naturlehre als in der Kunst hervorbringen“. ${ }^{110}$ Der Grundgedanke dieser geistigen Neuausrichtung ist - nun wird die Tragweite der in Italien angestellten Überlegung zur Kultur deutlich -, dass Beobachter und Phänomene, der Forscher und die zu erforschende Natur ineinander verwoben sind: „alles in der Natur [...] [ist] in einer ewigen Wirkung und Gegenwirkung.“111

Damit verschieben sich auch die Begriffe von Experiment und Experimentieren bzw. werden ineinander integriert. Das Experiment ist nicht mehr ein bloßes Instrument zur Erzeugung von Wissen, dem bei der Darstellung dieses Wissens keinerlei Bedeutung zukommt, sondern es ist das Erarbeiten einer Methode, die Beobachtung und Darstellung in einem ist. ${ }^{112}$ Goethe eröffnet damit nicht zuletzt auch ein anderes Verständnis für die Art und Weise der Entstehung von neuem Wissen. Die Gesetzmäßigkeiten der Farben ergeben sich bei Goethe deshalb aus den Beziehungen von Auge und Licht, also eigentlich aus einer dynamischen Wechselwirkung zwischen Sehsinn und Lichtquelle. In seinem Erkenntnismodel sind Beobachtung und Gegenstand nicht gesondert, sondern gehen ineinander über, durchdringen sich sogar auf das innigste, so dass die Anschauung selbst eine Reflexion, das Denken eine Beobachtung ist. Das entspricht zum einen einer Beobachtung der zweiten Ordnung: Erst aus der reflektierten Beobachtung, dem Hinterfragen von Beobachtungen lassen sich Erkenntnisse ziehen. Und zum anderen wird damit eine Epistemologie gefordert, die sinnliche Anschauung und

beurteilt. Erst als gegen Ende des 19. Jahrhunderts die mechanischen Erklärungsideale brüchig werden, erkennen Fachleute wie etwa Hermann von Helmholz die Fortschrittlichkeit von Goethes interdisziplinärer und ausdifferenzierter Wissenschaftspraxis, vgl. Carl Friedrich von Weizsäcker: „Einige Begriffe aus Goethes Naturwissenschaft". In: HA 13. S. 539-555 und Krohn: Goethes Versuch über den Versuch. S. 399-413, hier S. 401.

110 Goethe an Reichardt, Brief vom 30.5.1795, zitiert nach MA 4.2. S. 1020.

111 MA 4.2. S. 328.

112 Vgl. Krohn: Goethes Versuch über den Versuch. S. 399-413, hier S. 404. 
begriffliches Denken, konkretes Einzelnes und abstraktes Ganzes vermittelt. ${ }^{113}$ Goethe drängt in seinen Ausführungen stets auf die sinnliche Differenzierbarkeit der einzelnen Erscheinungen hin: Erfahrungen sollen mit den Sinnen - vor allem dem Sehsinn - beobachtbar sein, so dass sich an ihnen die Regelhaftigkeit ihrer Verwandlung augenfällig nachvollziehenlässt. ${ }^{114}$ In dieser Vorstelllung von Erkenntnis offenbart sich das, was wesentlich und wahr ist das Allgemeine, der Ursprung - dem Betrachter als unmittelbare Anschauung, anstatt als geistige Abstraktion. Die rationalistische Logik wird insofern in ihre enge Grenzen verwiesen ${ }^{115}$ und gleichzeitig die Ästhetik im Sinne von sinnlicher Wahrnehmung (aisthesis) als eigenständiges Wissenssystem stark gemacht ${ }^{116}$. Damit wird ein anderes Verständnis von Versuch und Wirklichkeit eröffnet, das nicht mehr auf die empirisch-normativen Ideale wie etwa Linnés Gattungshierarchie zurückgeführt werden kann, sondern korrektiv darauf einwirkt und sinnlich-intuitive Strategien begünstigt.

\subsubsection{Synergie zwischen Wissenschaft und Ästhetik}

Goethes Epistemologie stellt eine Versuchsanlage vor, die mehr auf das sinnfällige Zusammenstellen von vielfältigen und unterschiedlichen Resultaten bzw. „Erfahrungen" ${ }^{117}$ setzt als ein theoriegeleitetes, alleinstehendes experimentum crucis und vermag damit gerade auf dessen Schwächen hinzuweisen. Kants Vorgabe weiß nämlich mit den feinen, graduellen Unterschieden nicht umzugehen und überführt komplexe Gestalteigenschaften gezwungenermaßen stets in einen starren Dualismus. Dagegen entwirft Goethe im Grunde eine wirklichkeitsoffene Phänomenforschung, die auf das unvoreingenommene Erleben der Wirklichkeit setzt. Anstelle kontrollierender Vorannahmen und künstlicher Laborbedingungen fordert er die Bereitschaft, auf Unvorhergesehens, Überraschendes und die vielfältigen Wechselwirkungen dazwischen zu achten. Damit scheint die Morphologie eine überzeugende Antwort auf ein spezifisches Problembewusstsein der Moderne, die Fragmentierung des Wissens und der Lebenswelt geben zu können.118 Anstatt einzelwissenschaftlicher

113 Vgl. Maatsch: Morphologie und Moderne. Einleitung. S. 1-15, hier S. 6.

114 Dazu, wie zentral Sinnlichkeit für Goethes Gestalt-Begriff und eben auch DarstellungsBegriff ist, vgl. Biere, Florentine: Das andere Erzählen. Zur Poetik der Novelle 180o/19oo. S. 122-138; allg. zu Sinnlichkeit bei Goethe vgl. Naumann-Beyer, Waltraud: Sinnlichkeit. In: Witte; Janssen et al. (Hrsg.): Goethe Handbuch in vier Bänden. S. 987-989.

115 Inwiefern Kants Vernunftbegriff nicht leisten kann, worum es Goethe geht, vgl. Maatsch: Morphologie und Moderne. Einleitung. S. 1-15, hier S. 4.

116 Vgl. Schneider: Die Laokoon-Debatte: Kunstreflexion und Medienkonkurrenz im 18. Jahrhundert. S. $68-85$.

117 MA 4.2. S. $268 \mathrm{f}$.

118 Vgl. Maatsch: Morphologie und Moderne. Einleitung. S. 1-15, hier S. 10. 
Verzeichnung und Klassifizierung ermöglicht die explorative Epistemologie, Wissensfragmente zu sammeln und dadurch einen Überblick über Abhängigkeiten und strukturelle Analogien zu schaffen, sowohl innerhalb einzelner wie zwischen verschiedenen Wissensgebieten. ${ }^{119}$

Freilich können die an der organischen Natur entworfene Morphologie, die explorative Epistemologie und der sinnliche Erkenntnismodus nicht ohne weiteres auf literaturästhetische oder kulturphilosophische Probleme übertragen werden. Es lassen sich aber sehr wohl epistemologische Gewinne und Problemstellungen, die sich Goethe in den naturwissenschaftlichen Schriften offenbaren, gerade auch in seinen gattungstheoretischen Bemühungen nachweisen. ${ }^{120}$ Während die hohen, rigiden Ansprüchen auf Reinheit und Einheit der Gattungen ebenso fragwürdig geworden sind wie überhaupt eine theoriegeleitete Gattungsdefinition, erlauben das morphologische Formdenken und die Methodologie der Exploration, auch die ästhetischen Formen als variable, unabgeschlossene und heterogene Phänomene zu beschreiben, die offen sind für Interpretation und Veränderung. In dieser alternativen Wissensorganisation werden die einzelnen Formen auch nicht in eine hierachisch-chronlogische Systematik gezwungen, sondern können in diskursiven Formationen nebeneinander gestellt werden. Die Formen können so miteinander verglichen werden, ohne dass Unterschiede automatisch zu Werturteilen führen, Veränderungen innerhalb einer Gattung können gleichwohl sichtbar gemacht werden wie strukturelle Analogien zwischen verschiedenen Gattungen.

Darauf, wie ausschlaggebend das Experiment für Goethes literarische Produktion überhaupt, aber besonders für die in die Krise geratenen Formen des Erzählens ist, hat bereits Florentine Biere aufmerksam gemacht. Goethes Novelle weist sie den Status einer Anti-Gattung zu, die normative Vorgaben nicht befolgt, sondern vielmehr hinterfragt, sogar offen gegen sie verstösst und dadurch die genuin moderne Problemstellung reflektiert, etwas systematisch zu beschreiben, was sich festen Zuschreibungen gerade zu entziehen sucht. ${ }^{121}$ Dass Goethe sich diese Lizenzen des Experimentierens in besonderem Maße auch für die epische Produktion zunutze macht, hat sich insofern bereits abgezeichnet, als an seinen frühen Epos-Projekten ein bewusst spielerischer und kritischer Umgang mit den Gattungstraditionen nachgewiesen werden

\footnotetext{
119 Vgl. ebd. S. 1.

120 Zum Verhältnis von Literatur und Experiment im Allgemeinen vgl. Gamper, Michael (Hrsg.): Experiment und Literatur: Themen, Methoden, Theorien. Göttingen: Wallstein 2010; darin insb. ders.; Weder, Christine: Gattungsexperimente. Explorative Wissenspoetik und literarische Form: Aphorismus/Fragment/Notat - Essay - Novelle/Roman Lyrik (Gamper) - Märchen (Weder). S. 96-180.

Biere: Das andere Erzählen. Zur Poetik der Novelle 180o/19oo. S. 10-13.
} 
konnte. ${ }^{122}$ Weiter kann Goethes Selbstaussage, sich nun, nachdem Wolf das Epos als teilbar aufgezeigt hat, im epischen „Fache versuchen“ zu wollen, ${ }^{123}$ auf dem Hintergrund seines Wissenschaftsverständnisses als Hinweis darauf gelesen werden, dass es sich bei seinen epischen Arbeiten wohl um bewusste Experimente im Sinne seiner Versuchsanlage handelt. Die Bemühung, das Experiment als legitime Form des Unfertigen für die Ästhetik, die Kunst fruchtbar zu machen, steht zweifelsfrei in Zusammenhang mit Goethes Bewusstsein für den Traditionsbruch der Moderne. In gewisser Weise reflektiert er damit nämlich schon in den 179oer Jahren, was Nietzsche dann 1878 als das Hauptproblem der modernen Dichtkunst diagnostizieren wird: „Auch der Begabteste [gemeint ist Goethe] bringt es nur zu einem fortwährenden Experimentieren, wenn der Faden der Entwicklung einmal abgerissen ist. ${ }^{\prime 124}$ Während in Nietzsches Aussage deutlich der hegelsche Kulturpessimismus anklingt und ein pejorativer Experimentbegriff sichtbar wird, manifestiert sich in Goethes explorativer Versuchsanlage aber eine bedeutend optimistischere Perspektive. Ein fortwährendes Experimentieren, wie es im Aufsatz über den Versuch vorgestellt wird, eröffnet auch für die poetologische Produktion neue, potente Möglichkeiten, über den erlebten Bruch hinaus an die Tradition anzuschließen. ${ }^{125}$ Im Gegensatz zur normativen Gattungspoetologie, die den modernen Formerscheinungen nicht mehr gerecht wird und gerade im Fall des Epos nicht über die Feststellung des Gattungstodes hinaus kommt, erweisen sich die alternativen Beschreibungs- und Verfahrensweisen der Exploration und der Morphologie - Wiederholen, Ausbreiten, Sammeln und Vergleichen als vielversprechende Strategien, um die gefährdete Form des Epischen in die Moderne zu retten.

Unter diesem Aspekt bildet der Rückgriff auf die antike Form des Epos sprich der Wiederholungsversuch, im Sinne eines wieder holens - zwar immer noch den Kern der epischen Produktivität, erhebt aber nicht mehr den Anspruch auf eine mimetische Nachbildung. Goethes epische Werke werden

122 Vgl. S. 55 ff. in diesem Buch.

123 Goethe an Wolf, 26.12.1796, WA 11. S. 296.

124 Nietzsche, Friedrich:Die Revolution in der Poesie, zitiert nach Keller:Die ungeschriebenen Propyläen - Klassizismus im Experiment. S. 387-407, hier S. 390 f. Nietzsche konstatiert wie Hegel einen Abbruch der Tradition, macht aber namentlich Lessing für den spezifischen Abbruch der, stetigen Befreiung der Dichtkunst‘ aus den alten Fesseln verantwortlich.

125 Vgl. Schneider, Sabine: Das sentimentalische Spiel mit den Archiven des kulturellen Gedächtnisses. Schillers ludistische Ästhetik als Reflexion auf die Bedingungen künstlerischer Produktivität am Beginn der Moderne. In: Alt, Peter-André; Lepper, Marcel; Raulff, Ulrich (Hrsg.): Schiller, der Spieler. Göttingen: Wallstein 2013. S. 242-261, hier S. 242. 
sich insofern nicht als normkonforme Epen erweisen, sondern als gezielte Form-Experimente im Spannungsfeld von Roman und Novelle, Idylle und Epos, die sich gerade durch das Überschreiten konservativer Gattungsgrenzen auszeichnen. Voraussetzung für eine derartige Hybdridisierung von verschiedenen Gattungstraditionen über ihre materiellen Grenzen hinaus ist, das Gattungssystem als beweglichen, lebendigen Wissenszusammenhang zu betrachten. Darin besteht das besondere Verdienst der Morphologie für Goethes poetologisches Formdenken überhaupt und insbesondere für sein Epos-Projekt, wie sich im Weiteren noch genauer zeigen wird. 